\title{
DATA REPORT FOR A SEISMIC REFRACTION/ WIDE-ANGLE REFLECTION INVESTIGATION OF THE ATLANTIC COASTAL PLAIN IN SOUTH CAROLINA
}

By

James Luetgert, Harley Benz, Ed Criley, and Li Song-Lin '

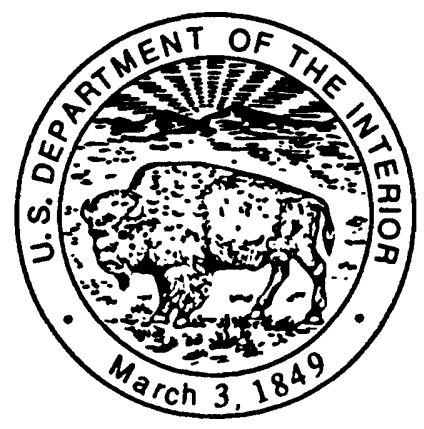

OPEN-FILE REPORT $92-723$

This report is preliminary and has not been reviewed for conformity with

U.S. Geological Survey edltorlal standards or with the North American Stratigraphic Code. Any use of trade, product or firm names ls for descriptive purposes only and does not Imply endoreement by the U.S. or Canadian Governments. 
TABLE OF CONTENTS

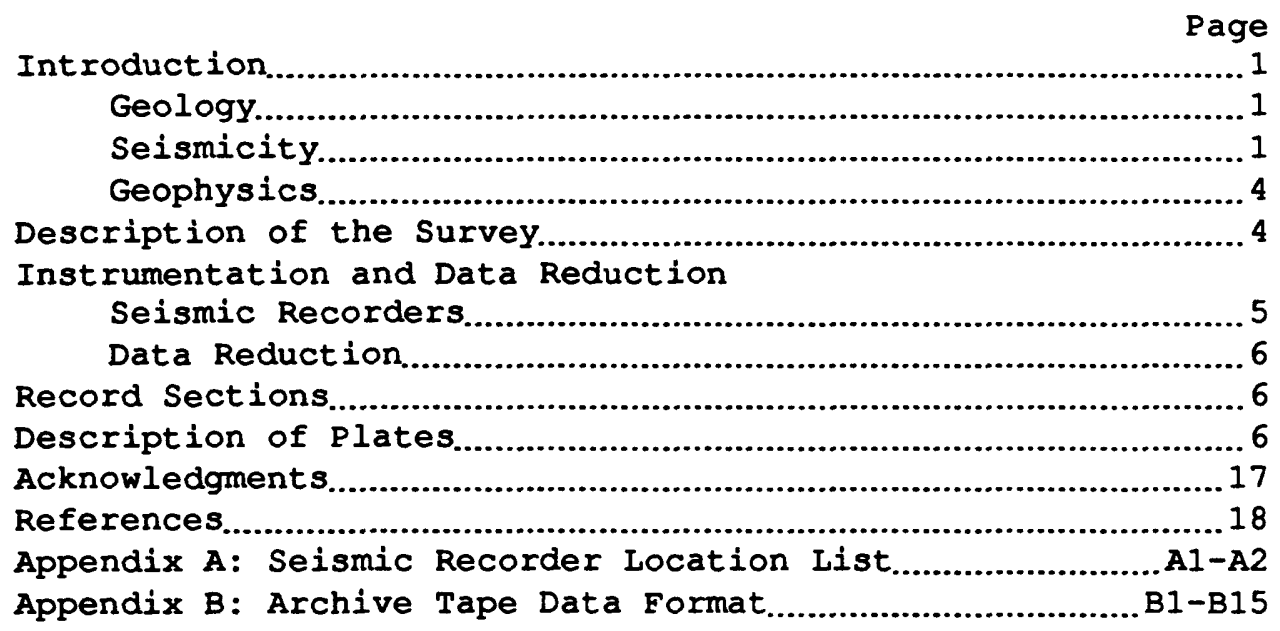

FIGURES

1. Location Map of Study Area................................................... 2

2. Basement contours...................................................................... ${ }^{3}$

3. Record Section, Shotpoint 1 - vertical................................7

4. Record Section, Shotpoint 2 - vertical.............................. 8

5. Record Section, Shotpoint 3 - vertical............................. 9

6. Record Section, Shotpoint 4 - vertical...............................10

7. Record Section, Shotpoint 5 - vertical..............................11

8. Record Section, Shotpoint 1 - horizontal...........................12

9. Record Section, Shotpoint 2 - horizontal..........................13

10. Record Section, Shotpoint 3 - horizontal.......................... 14

11. Record Section, Shotpoint 4 - horizontal..........................15

12. Record Section, Shotpoint 5 - horizontal.........................16

\section{TABLES}

1. Master Shot List. .5 


\section{Intreduction}

In April, 1991, the U.S. Geological Survey (USGS), in collaboration with the Department of Energy (DOE) and Savannah River Site (SRS), conducted a seismic refraction/wide angle reflection experiment in the Atlantic Coastal Plain region of South Carolina. The profile line extended east from New Ellenton, SC to Walterboro, SC crossing a region characterized by early Mesozoic rifting and sedimentation associated with the opening of the Atlantic ocean. Portable vertical seismographs were located along this line at intervals of $1000 \mathrm{~m}$. Portable horizontal seismographs were co-located with every second vertical seismograph along this line at intervals of $2000 \mathrm{~m}$. Five shotpoints were located at intervals of $30 \mathrm{~km}$.

This report is a compilation of the data collected by the USGS. The data have been archived at the National Geophysical Data Center in Boulder, Colorado. Tapes are available from:

\section{U.S. Department of Commerce}

National Oceanic and Atmospheric Administration

325 Broadway

Boulder, $\mathrm{CO} 80303$

(303) $497-6472$

Appendix B contains a description of the tape format. Interpretations of these data will be published separately.

\section{Geology}

The seismic refraction/wide-angle reflection profile is located on the Atlantic Coastal Plain of South Carolina (Figure 1). At this latitude, the Coastal Plain is about $200 \mathrm{~km}$ wide with a NE-SW strike along the coast. To the northwest is the Appalachian Fold Belt. To the southeast there exist two offshore parallel belts, the Carolina Platform and Carolina Trough which form the transitional zone between the North American Continent and the Atlantic Ocean.

The Atlantic Coastal Plain is covered by Mesozoic and younger marine deposits, which lie unconformably upon Jurassic basement. The basement surface generally dips gently to the southeast. Figure 2 shows the contours of the basement surface (Maher, 1971). Along the profile, the upper sedimentary layer is $300-800$ meters thick.

\section{Seismicity}

Moderate seismicity occurs in many parts of south Carolina. In the Piedmont and Upper Atlantic Coastal Plain, localized reservoir-induced seismicity is superimposed on a diffuse lower frequency background. In contrast, moderate seismicity in the middle and lower Coastal plain region occurs within discrete seismogenic zones. The Coastal Plain region of south Carolina is important, since evidence for historic and prehistoric large earthquakes is now available. The most recent large earthquake is the 1886 event near Charleston $(M=7.6)$. 


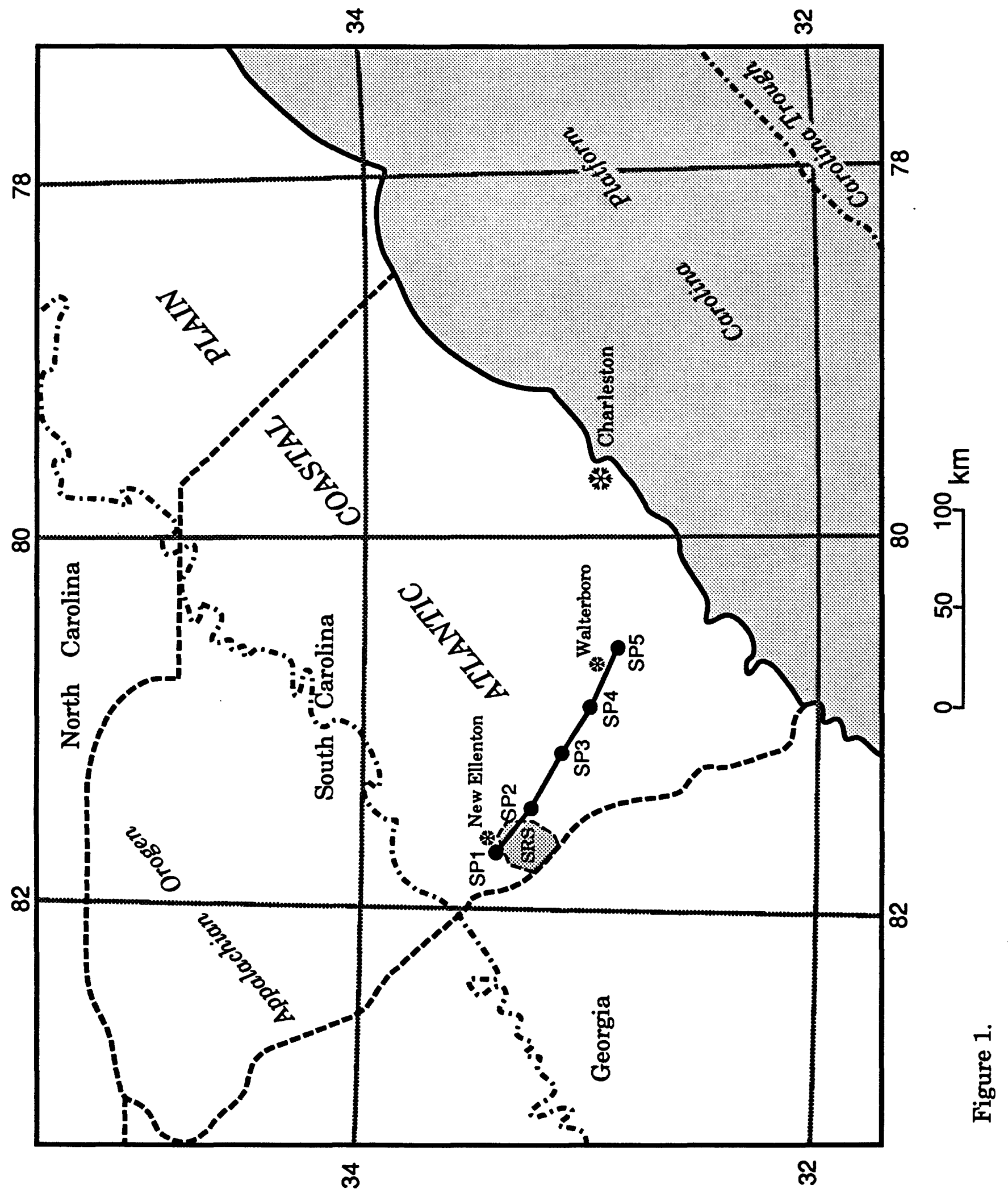




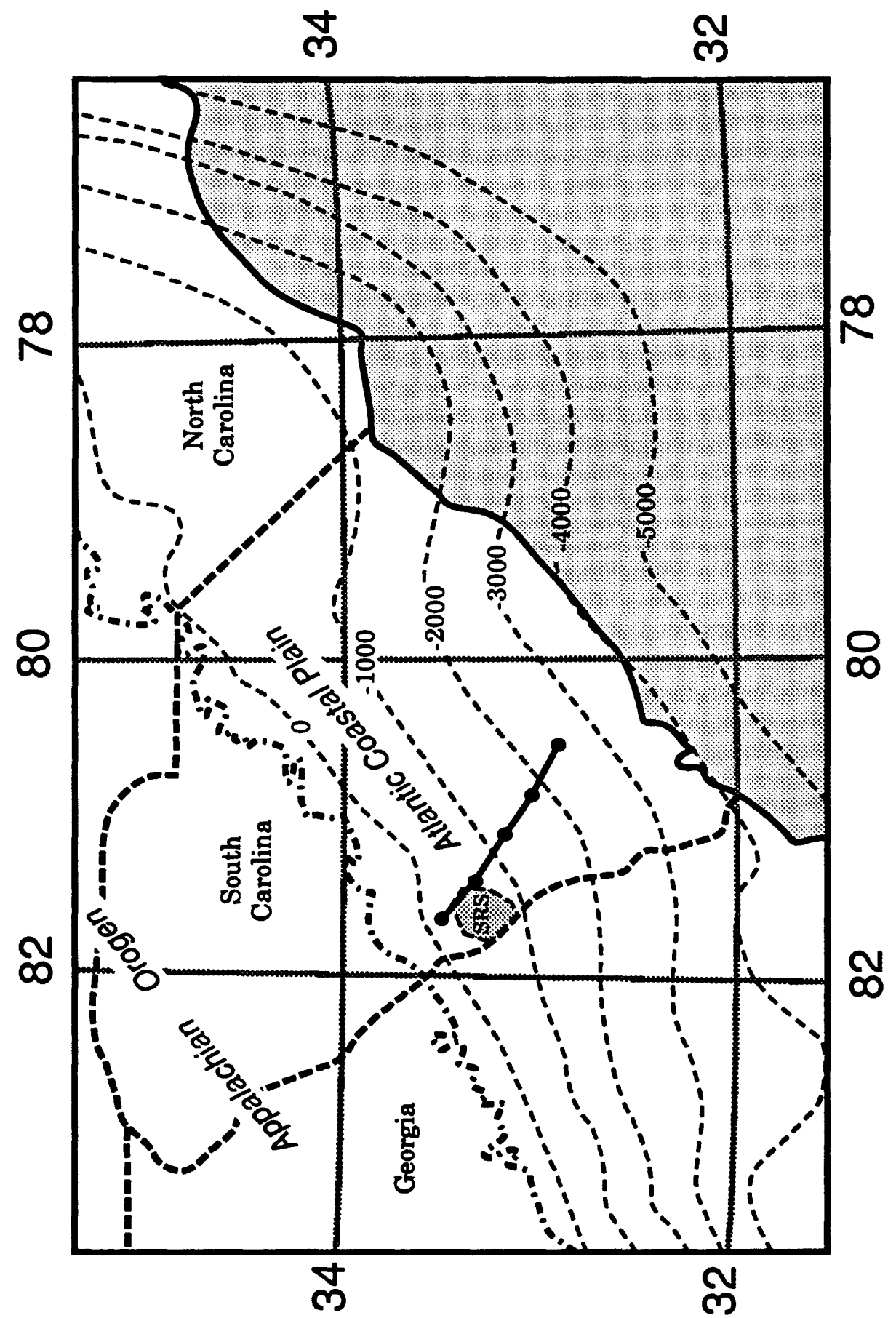

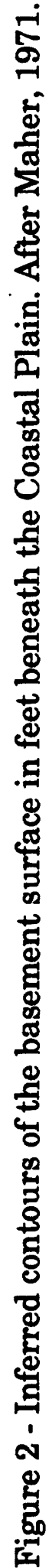


Open File $92-723$

\section{Geophysics}

The tectonic framework below the Coastal Plain sediments has been studied by a number of geophysical surveys carried out over the last several years. Earlier refraction studies by Bonini and Woollard (1960) provided information about the $\mathrm{P}$-wave velocities in the basement. Reinterpretation of these data by Daniels (1974) suggested the presence of granite, mafic gneiss/schist, Triassic sediments, and basalts where Bonini and Woollard interpreted the Carolina slate belt rocks. Seismic refraction and reflection studies in the area of Charleston, SC, (e.g. Ackermann, 1983; Hamilton, et al., 1983) provide information about the seismic velocities and structures in the post-Jurassic upper crust. Interpretation of COCORP seismic reflection data by Cook et al., (1981) argues that the lower Paleozoic decollement continues below the Coastal Plain sediments.

Well data within the SRS and surrounding regions provide additional constraints on the velocities and lithologies of the basement rocks, wherever encountered. Regional gravity and aeromagnetic studies in the region (Popenoe and Zietz, 1977, Daniels and zietz, 1978, and Daniels et al., 1983) identified long wavelength anomalies which are caused by Mesozoic and older rocks below the coastal plain sediments.

\section{Description of the Survey}

Portable seismic recorders were laid out along the profile in a continuous linear pattern (Figure 1). Five shots were fired at 5 locations along the profile (shotpoints 1-5) (Table 1).

Instruments with vertical sensors were placed at a nominal spacing of 1000 meters. In addition, an instrument with a transverse horizontal sensor was co-located at every other site.

Recording instrument and shot point locations and elevations were determined using the satellite-based Global Positioning System (GPS). All the locations (Appendix $A$ ) are estimated to be accurate to within 2 meters; elevations within 5 meters.

Shotpoints 2, 3, and 4 were sited in $20 \mathrm{~cm} \mathrm{X} 45 \mathrm{~m}$ drill holes (Table 1). Ammonium nitrate explosive was detonated by electric detonators (caps), detonating cord, and boosters. Shotpoints 1 and 5 (Table 1) were sited in deep drill holes at depths of 260 and 800 meters respectively. The bottom 65 meters of SP1 were in crystalline rock. SP5 was in consolidated, but noncrystalline rock. In deep holes a special explosive and detonators are required to withstand the hydrostatic pressure. In this case, we used an $80 \%$ nitroglycerin product from IRECO called Gas Well Gelatin. The explosive is packaged in a hard cardboard sleeve, $12.7 \mathrm{~cm}$ in diameter, 3.2 meters long, weighing $45.4 \mathrm{~kg}$. From each end a wire loop extended for handling. Four detonators were used in each hole. Two for each end of the charge were connected in parallel, then connected to a 820 meter spool of assualt wire, the connection sealed with silicon gel and left overnight to cure. The wire of the spool is \#20 gauge consisting of 3 strands of copper wire and 5 strands of steel wire. Each 3.2 meter explosive cartridge was lifted with a a truck mounted derrick to a vertical position and lowered into the hole to a depth where the cable of the cartridge could be attached to the $.5 \mathrm{~cm}$ downline cable with small ' $U$ ' bolts. After the completion of attaching and lowering each of the 20 cartridges, the downline with attached cartridges was lowered to the bottom of the hole. The two cartridges with the detonators were placed 2 nd 
from the bottom and 2nd from the top of the column of explosives. Continuity of the shot lines with detonators was carefully monitored as they were fed downhole with the downline cable. One detonator and one shot line would have been sufficient to detonate the charge; the additional detonators and line were for backup. The shotlines were connected in parallel and shot with a 2000 volt blaster. All shots were fired automatically by portable master clocks calibrated to time obtained from the GOES satellite. The reported shot times are accurate to within \pm 2 milliseconds, assuming that the explosives detonated at the exact time of the cap break.

\section{Table 1}

\section{SHOT LIST}

\begin{tabular}{|c|c|c|c|c|c|c|c|c|c|}
\hline $\begin{array}{l}\text { Shot } \\
\text { No. }\end{array}$ & $\begin{array}{l}\text { Shot } \\
\text { Point }\end{array}$ & & te & $\begin{array}{c}\text { Shot Time } \\
\text { Day:Hr:Mn:Sec }\end{array}$ & $\begin{array}{l}\text { Size } \\
(\mathrm{kg}) \\
\end{array}$ & $\begin{array}{c}\text { Latitude } \\
\text { (deg) }\end{array}$ & $\begin{array}{c}\text { Longitude } \\
\text { (deg) }\end{array}$ & $\begin{array}{l}\text { Elev. } \\
\text { (m) }\end{array}$ & $\begin{array}{l}\text { Depth } \\
\text { (m) }\end{array}$ \\
\hline $\begin{array}{l}1 \\
2 \\
3 \\
4 \\
5\end{array}$ & $\begin{array}{l}1 \\
2 \\
3 \\
4 \\
5\end{array}$ & $\begin{array}{l}1991 \\
1991 \\
1991 \\
1991 \\
1991\end{array}$ & $\begin{array}{l}4 / 7 \\
4 / 7 \\
4 / 7 \\
4 / 7 \\
4 / 7\end{array}$ & $\begin{array}{l}97: 02: 00: 00.000 \\
97: 02: 02: 00.000 \\
97: 02: 04: 00.000 \\
97: 02: 06: 00.000 \\
97: 02: 08: 00.000\end{array}$ & $\begin{array}{l}997.9 \\
997.9 \\
997.9 \\
997.9 \\
997.9\end{array}$ & $\begin{array}{l}33.411393 \mathrm{~N} \\
33.267005 \mathrm{~N} \\
33.128413 \mathrm{~N} \\
33.006499 \mathrm{~N} \\
32.883426 \mathrm{~N}\end{array}$ & $\begin{array}{l}81.706984 W \\
81.440724 W \\
81.161885 W \\
80.919387 W \\
80.580914 W\end{array}$ & $\begin{array}{r}168 \\
97 \\
101 \\
49 \\
21\end{array}$ & $\begin{array}{r}260 \\
30 \\
30 \\
30 \\
800\end{array}$ \\
\hline
\end{tabular}

INSTRUMENTATION AND DATA REDUCTION

Seismic Recorders

The seismic group recorders used by the USGS in this seismic-refraction survey were 180 modified single-component SGR IIIs. The SGR-III is a single channel, digital seismic recorder with a theoretical dynamic range of $156 \mathrm{~dB}$. Data is sampled at 500 samples per second by a 12 bit $A / D$ with gain ranging from $0-90 \mathrm{~dB}$ in $6 \mathrm{~dB}$ steps. The instruments have been modified to turn on at preset times instead of using the standard radio turn on. A programmable memory board in each unit allows data to be recorded during 99 predetermined time windows. Timing is provided by a temperature compensated internal oscillator that is synchronized to a USGS master clock prior to deployment. The USGS master clocks drift approximately one millisecond per week and are checked periodically against satellite clocks. The digital data and a drift rate are recorded on cartridge tape. The drift rate is used to calculate a chronometer correction at shot time. The SGR-III recorders were designed by Amoco Production Company, built by Globe Universal Sciences, Inc., and modified by the USGS.

In this investigation, 120 instruments were connected to Mark Products L4A 2-Hz vertical-component geophones. 60 instruments were connected to Mark Products I4A 2-Hz horizontal-component geophones. Horizontal geophones were co-located with vertical geophones at every second recording location.

The clocks of each recording unit were initially synchronized to a GOES master clock via a portable base receiver. Each unit was then deployed with programmable timers to initiate recording over the expected shot time window. After the deployment the GOES time signal was compared to the internal clocks for drift measurement. All data were time corrected using the GOES data assuming a linear drift rate. 


\section{Data Reduction}

Following the experiment, data from all instruments was written in SEGY-LDS format and merged into shot gathers. All data have been resampled to 200 samples per second and header information has been checked for accuracy and consistency.

\section{Record Sections}

For each shot a trace-normalized record section is presented (Fig. 3-12).

All traces are normalized to their maximum deflection and plotted using reduced time, with a reduction velocity of $6.0 \mathrm{~km} / \mathrm{s}$ for record sections recorded by vertical sensors and with a reduction velocity of $3.46 \mathrm{~km} / \mathrm{s}$ for record sections recorded by horizontal sensors. All traces have been bandpass filtered from 2 to $20 \mathrm{~Hz}$ to attenuate high frequency noise bursts and ground roll. A few traces which recorded no data have been removed for clarity.

In order to make the record sections (Fig. 3-12) easier to analyze, a few traces were deleted in areas where a noisy trace obscured surrounding data.

\section{Description of the plates}

Figure 3 shotpoint 1 recorded by instruments using vertical sensors. Figure 4 shotpoint 2 recorded by instruments using vertical sensors. Figure 5 shotpoint 3 recorded by instruments using vertical sensors. Figure 6 Shotpoint 4 recorded by instruments using vertical sensors. Figure 7 Shotpoint 5 recorded by instruments using vertical sensors.

Figure 8 Shotpoint 1 recorded by instruments using horizontal sensors. Figure 9 Shotpoint 2 recorded by instruments using horizontal sensors. Figure 10 shotpoint 3 recorded by instruments using horizontal sensors. Figure 11 shotpoint 4 recorded by instruments using horizontal sensors. Figure 12 shotpoint 5 recorded by instruments using horizontal sensors. 


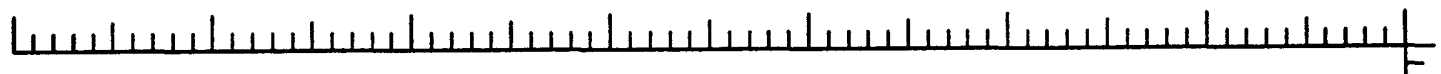

911 max 1) S 611 wint

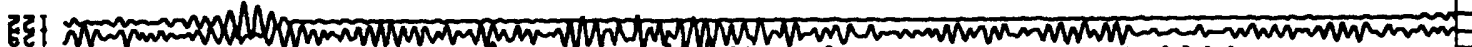

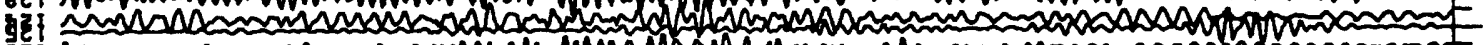

get

6 (1)

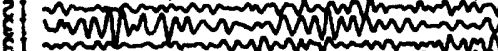

(

$\mathrm{E}_{\mathrm{E}}$

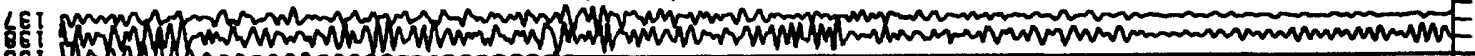

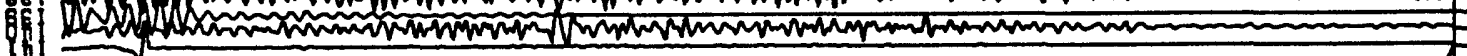

EH

If

It

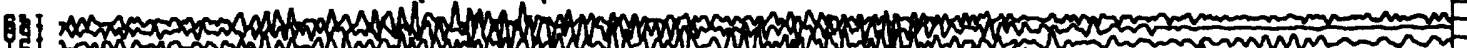

(5)

SIT.

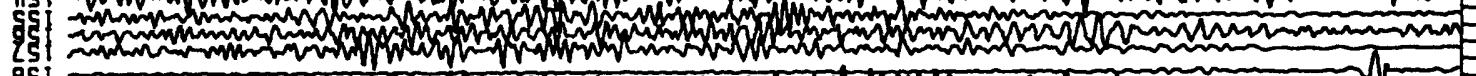

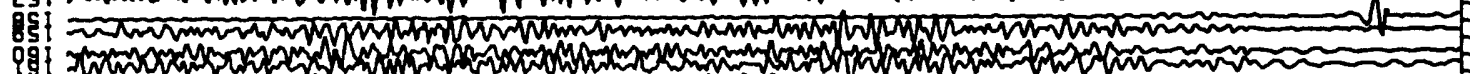

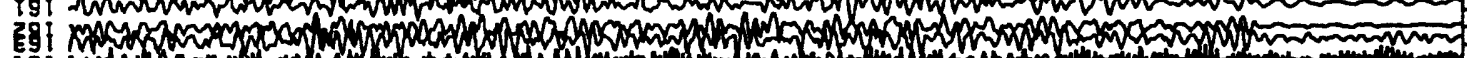

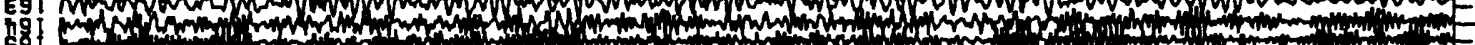

5.

III 6

5.1

65)

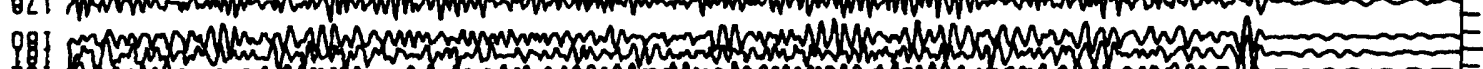
Hita

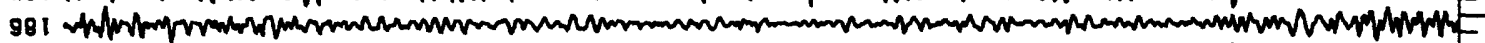

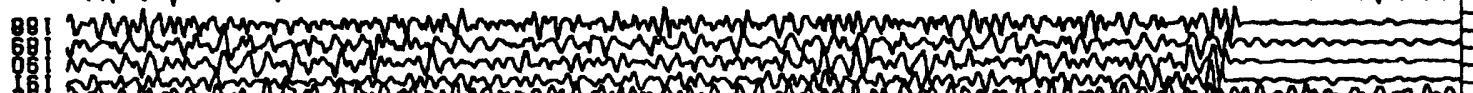

15.

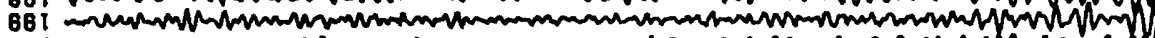

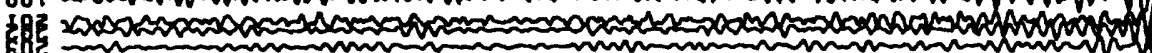

10

(2)

2

g

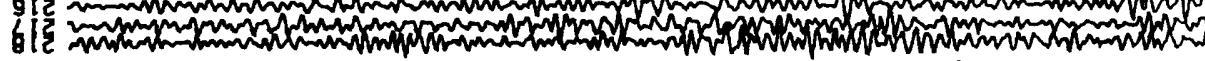

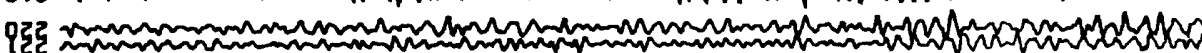

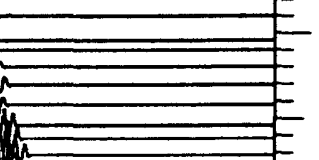

हEᄅ ₹

Gect

C

เ

$\exists$

$m$

त 

(n)

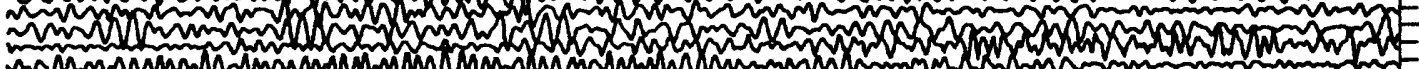
mind

2!

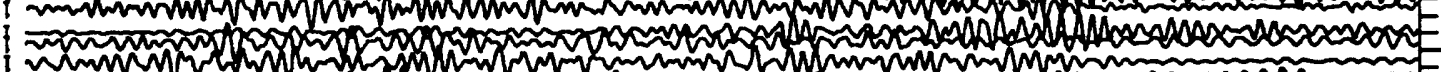
Din int mint xwe

6EI

(1)

E⿱⺈ Ima (n)

$14 A 4 \times A \circ D=200$

39.

(n)

P马i

E⿰冫.

(n)

191 ;

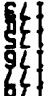

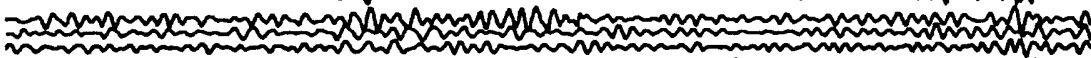

(1)

P:

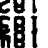

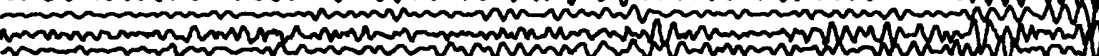

981 (1)

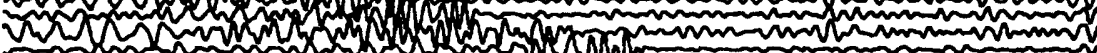

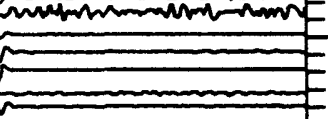

2

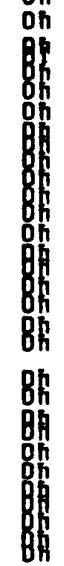

661

ב

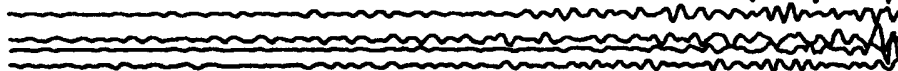

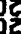

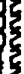

$\approx \approx-12010$

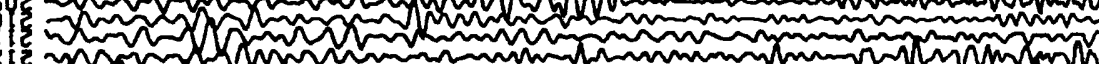

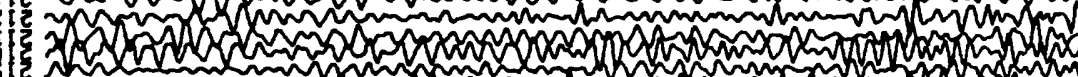

gle

s! 2010

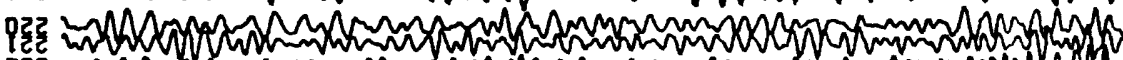

Ezᄅ

娓

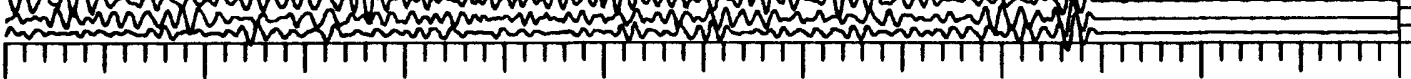

$(1$

บ

$\exists$

m

N

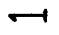

$\longrightarrow$

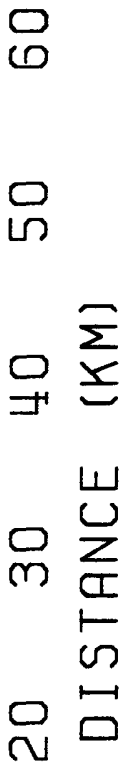




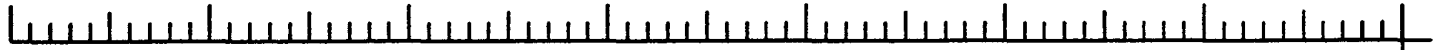

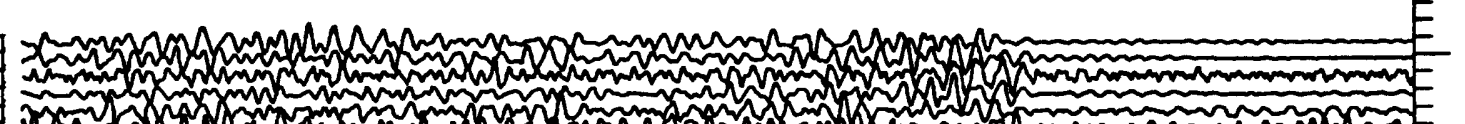
2) inl AMr

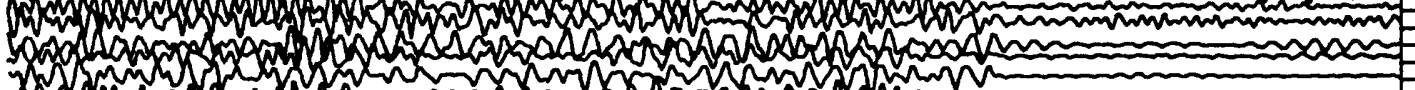
1) 21 10.4nz Q minn $=$ C.T. 斯;

g.t. $\rightarrow$ mon

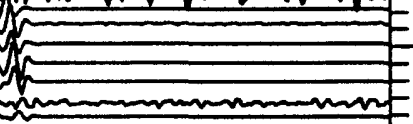

路

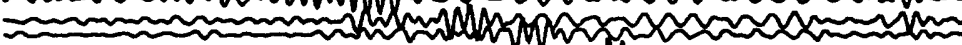

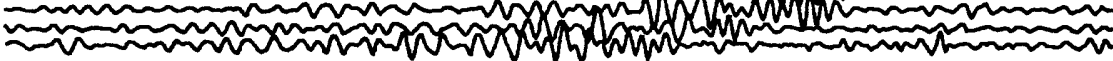

(1)

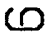

เ)

$\exists$

m

त

$\rightarrow$

$\square$

$\multimap$ 
0

m $¥ m$

N

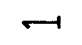

$\varnothing$

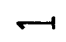


o

县

7.

然

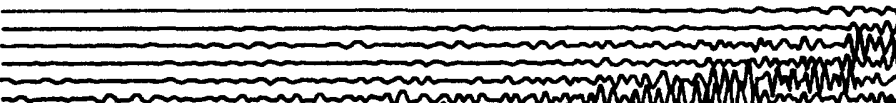

(1)

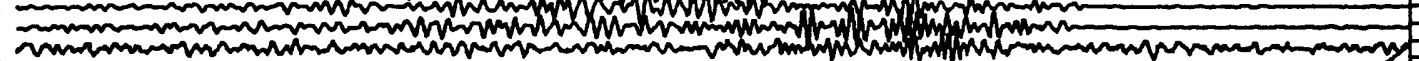

$\approx$

$\lim _{\min }$

miming

(1)

miman

(n)

(n)

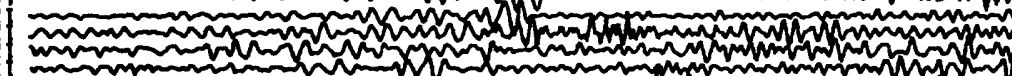

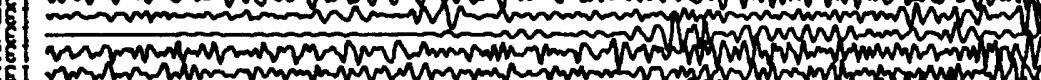

1

681 (n)

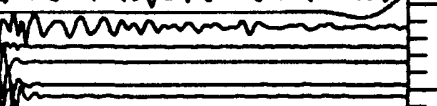

th

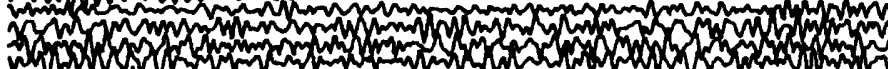

斯.

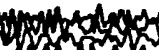

ș. (1) 2

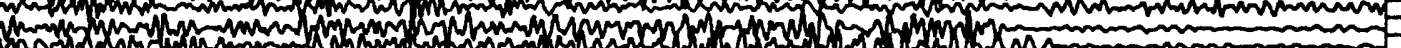

SEI

I

W

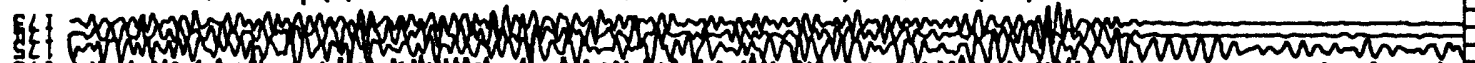

(1)

\&

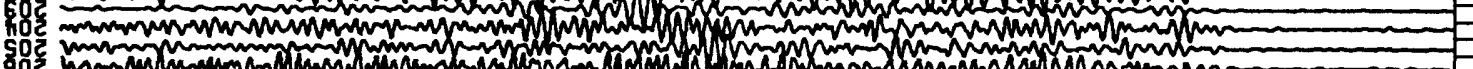

(1)

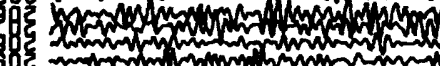

(1)

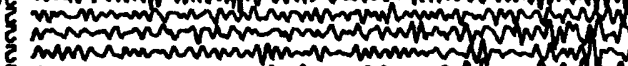

1

6)

$61 e^{2}$

P㞯

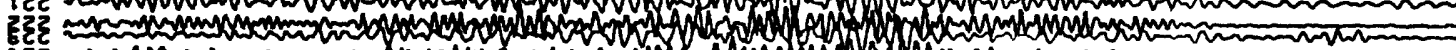

nez

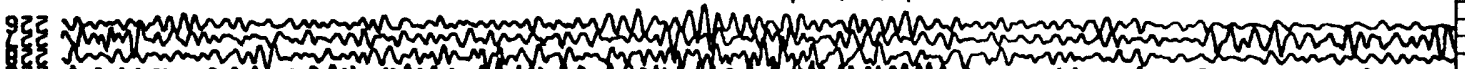

6 E

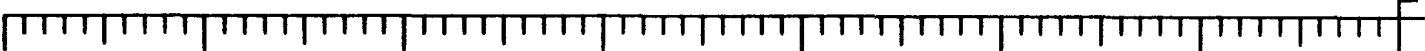

(0)
เ

$\exists$

m

त

$0 \cdot 9 / x-1$ $\therefore$

\&

宁

点

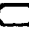

$m$

I

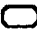

I

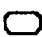

1 


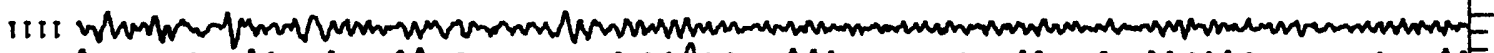

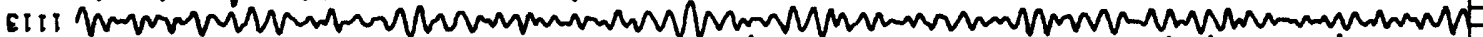

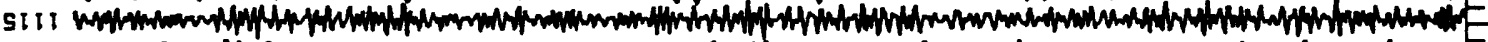

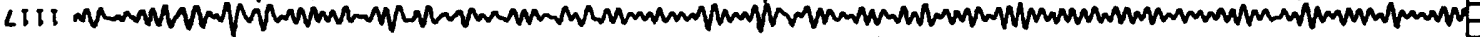

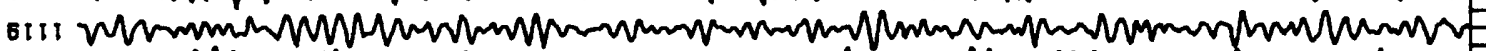

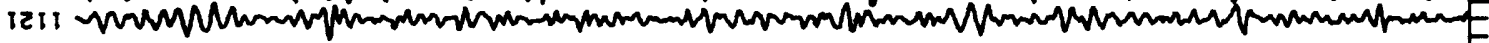

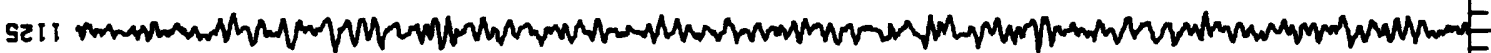

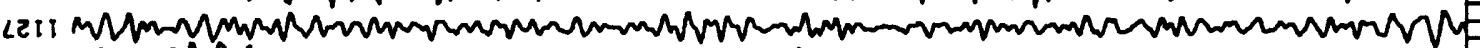

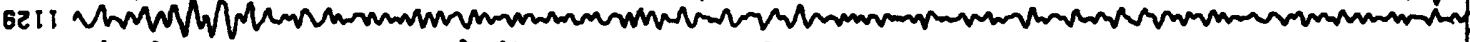
iE II MMW

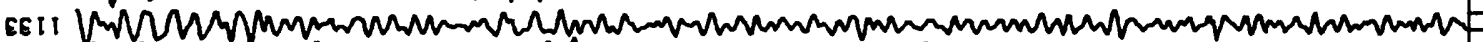

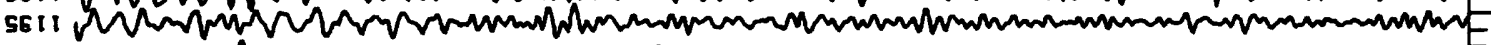
LE I 6E I; InIt

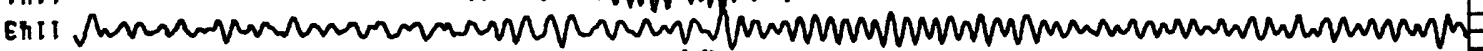
Shit

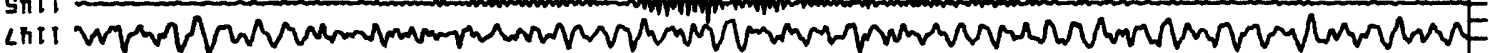

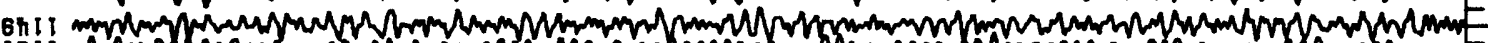

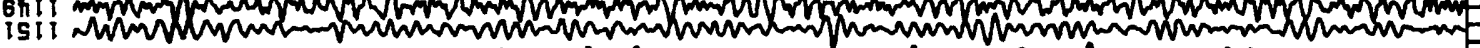

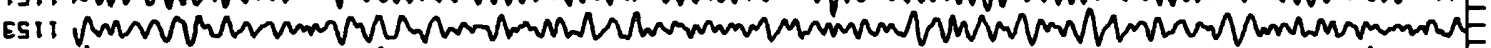

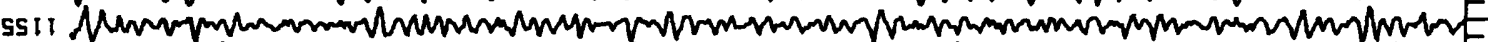

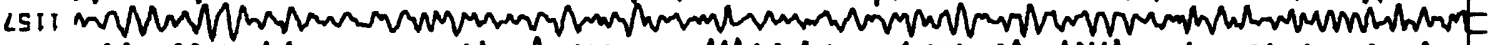

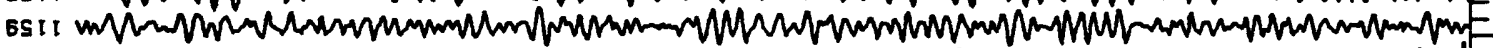
Ig I I r a

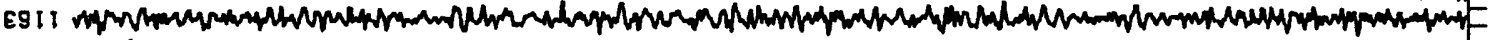
S9 II $\mathrm{WWWWW}$

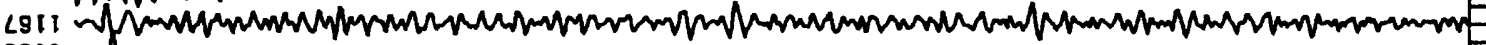

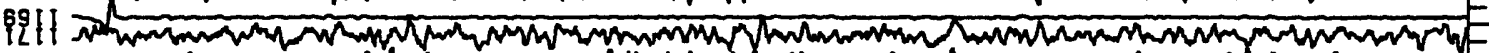

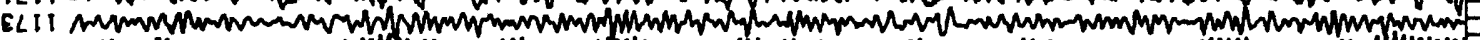

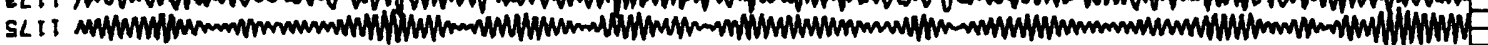

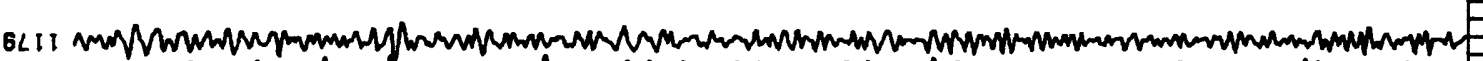

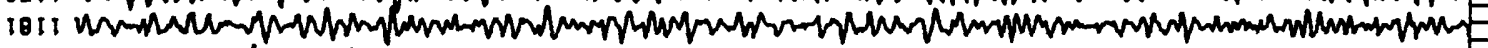

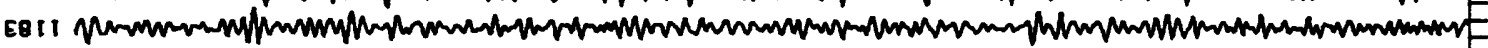

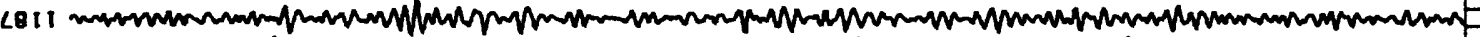

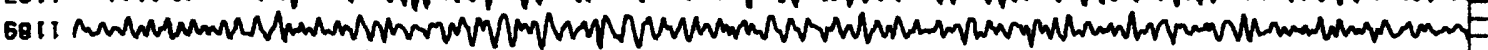

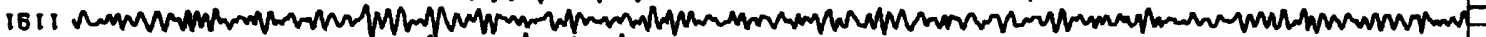

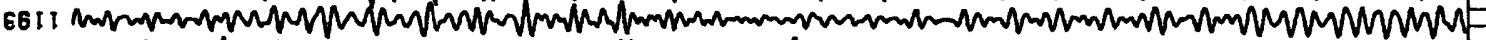

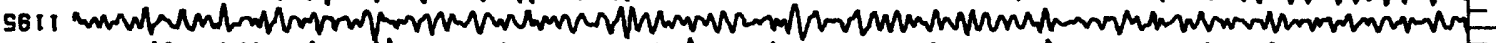

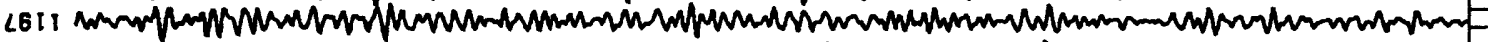

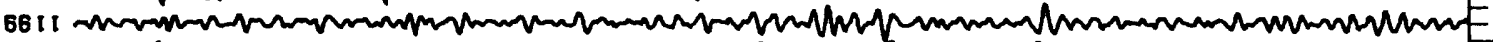

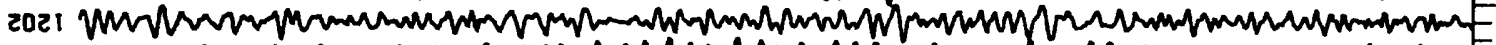

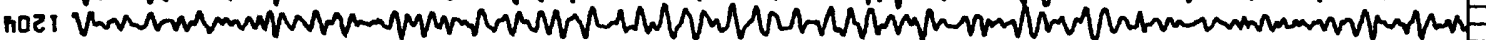

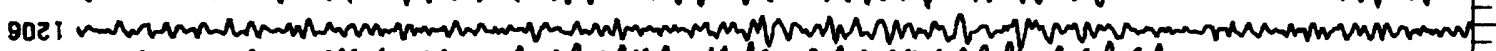

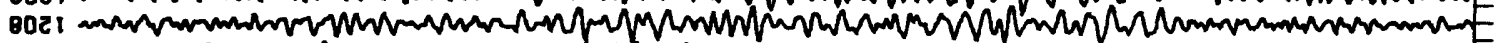

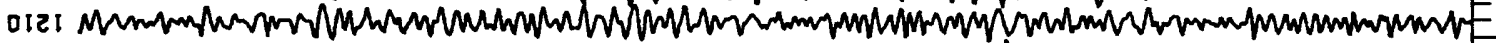

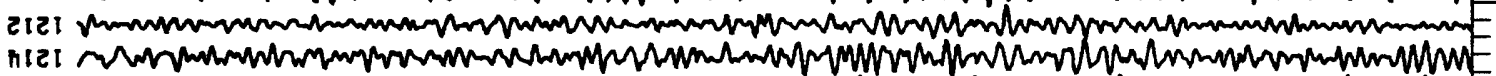
812 I QI II I 0221 M

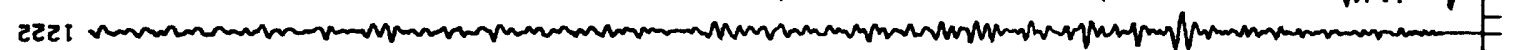

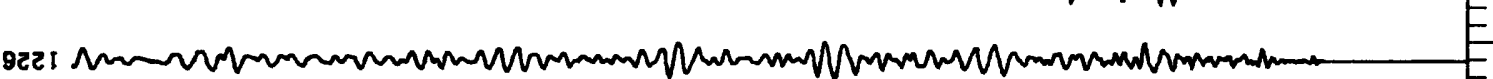

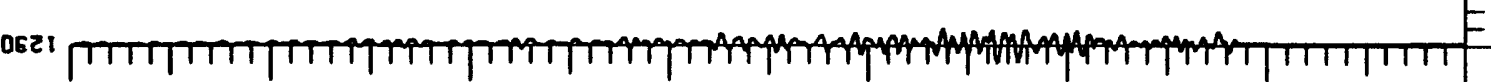


Oh
III

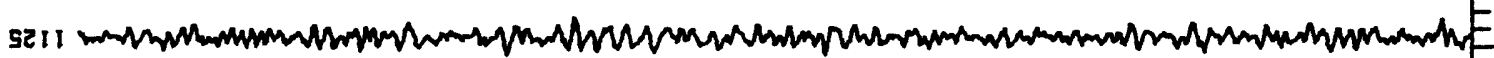
LEII MWWWMm

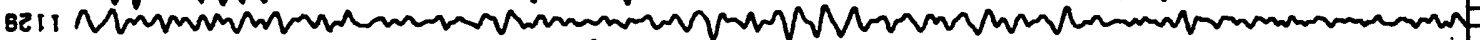

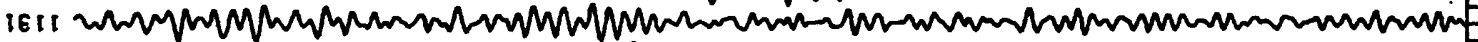

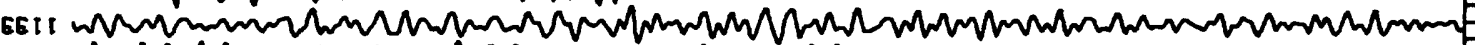

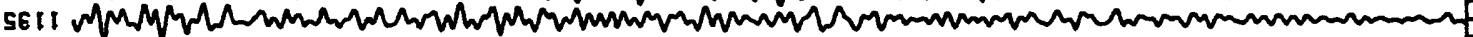
LEI I

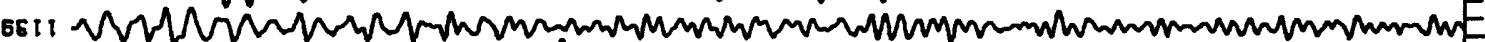
ItI En $\mathrm{WWH}$

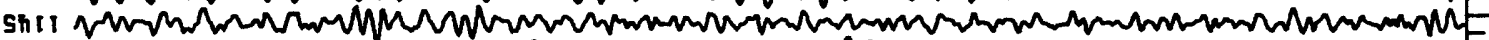
LII

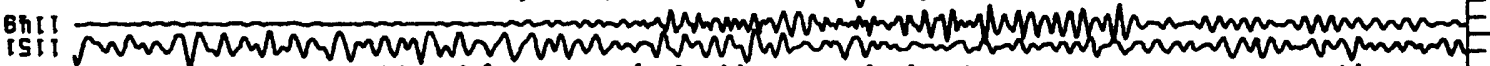
Es i

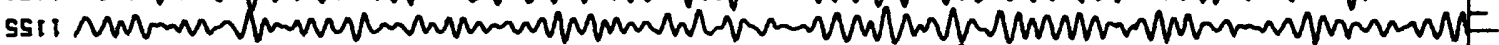

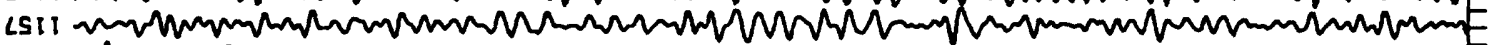

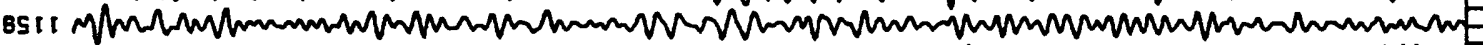
1911 m 11 mormon

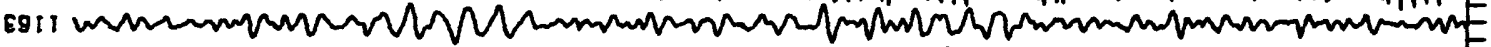

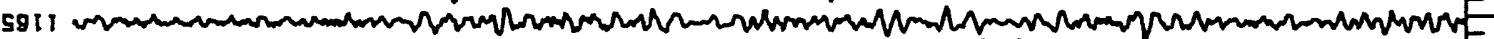

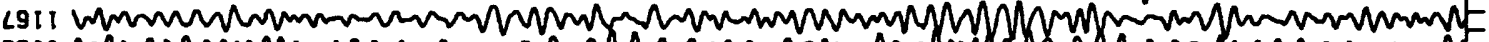
9211 xhax ELII

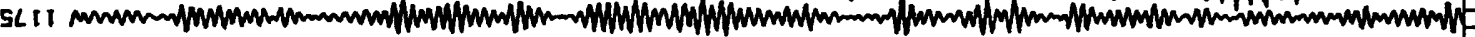

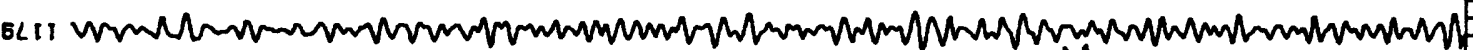

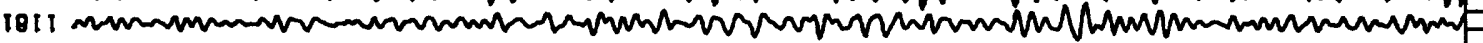

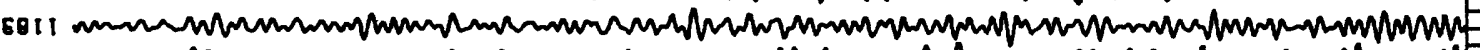

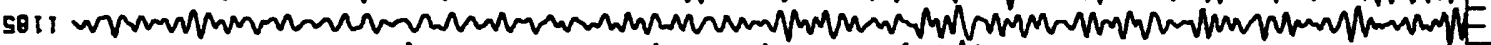

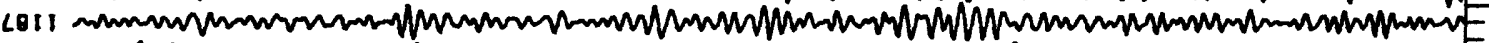

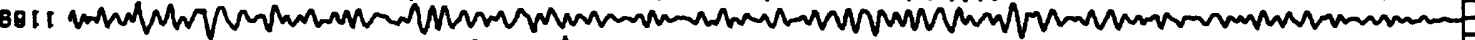

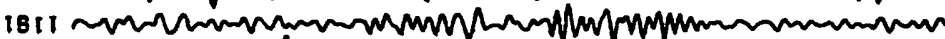

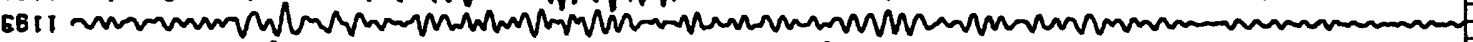
S6II CBIt C $66 \mathrm{tt}$ nozI

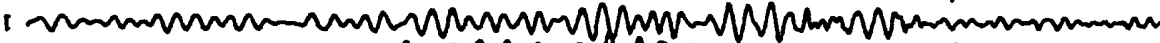
(n)

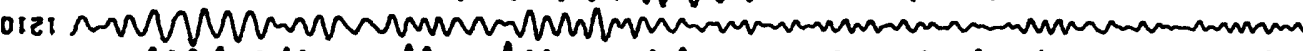
zाzi

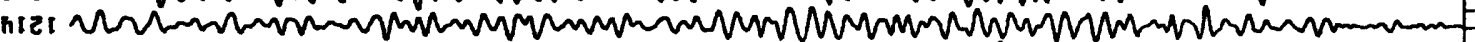

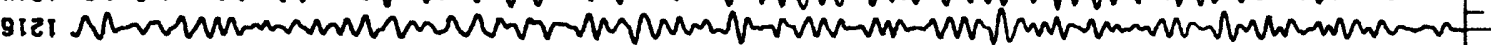
Q 2 i i

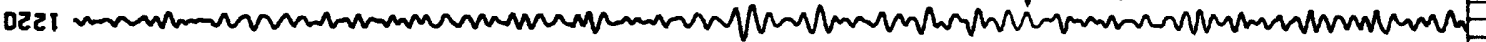

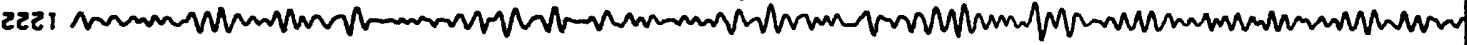

9281 OE2I

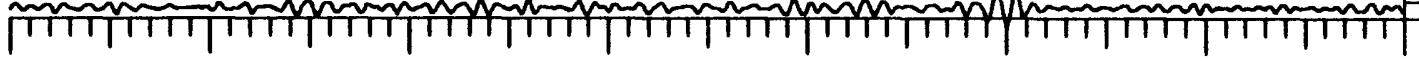
$(0$ เก $\exists$ $m$ $g \pi \cdot \varepsilon / X-1$ N $\longrightarrow$ 


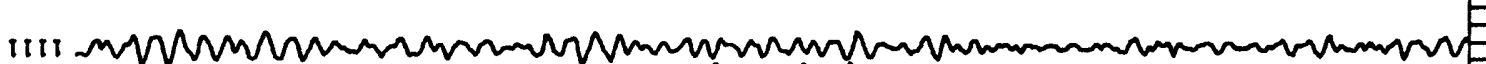

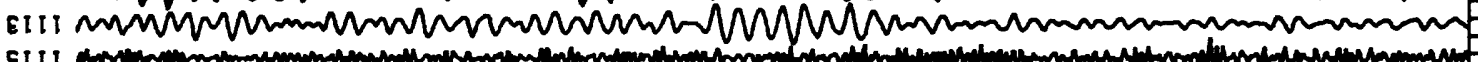

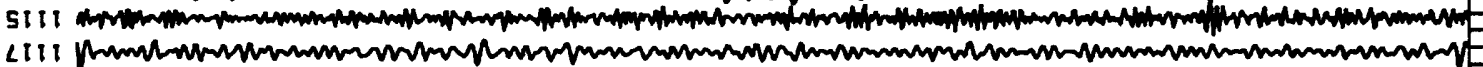

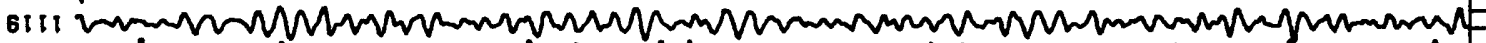

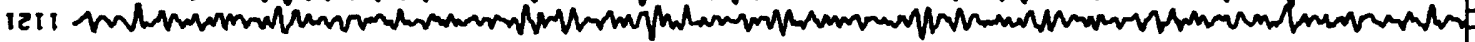

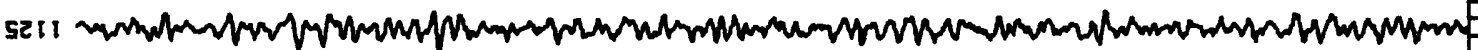

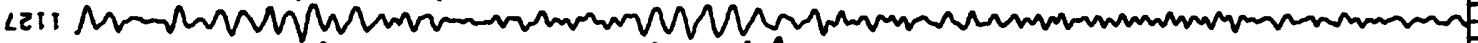
6eII

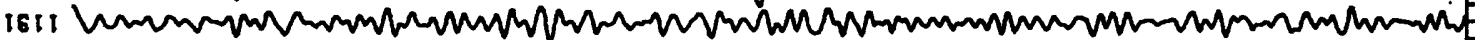

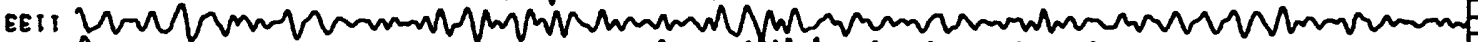

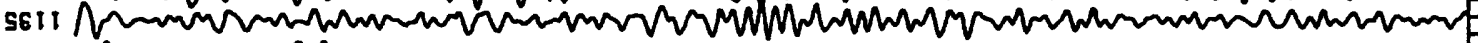

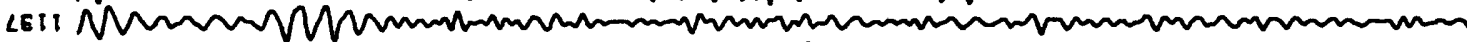
BEII

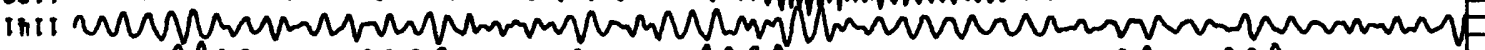

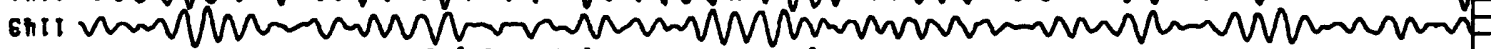

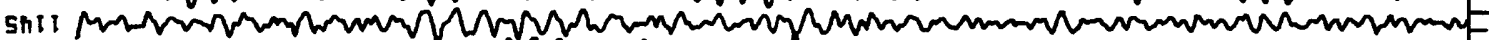

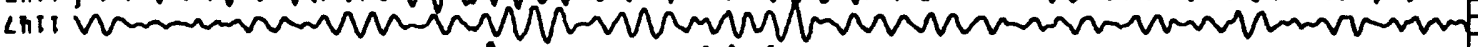
isti

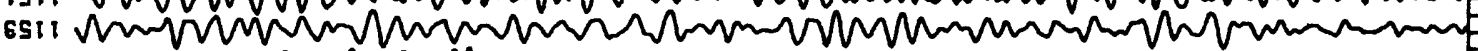

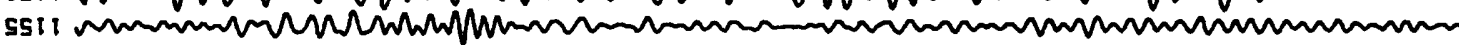

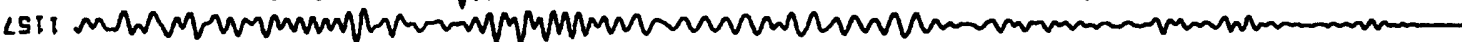
6SII 11 m 1911

0

บ

$\mathbf{I}$

$m$

N 
|

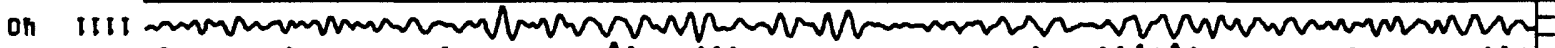

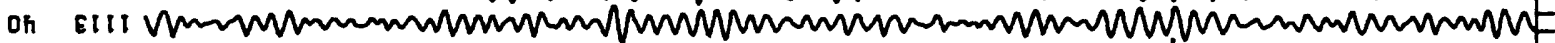

oh

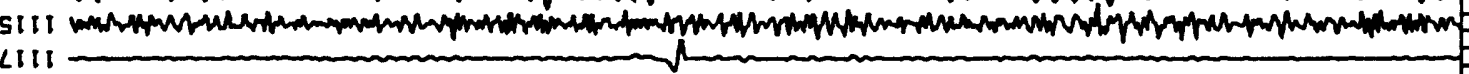
6tit

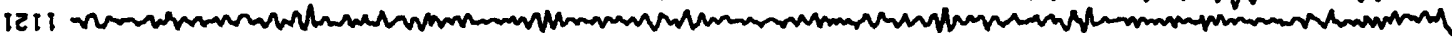

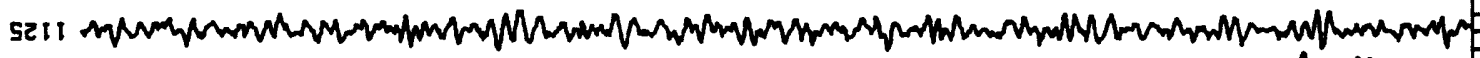
LetI 6etI IE 11 1 SEII II LEII

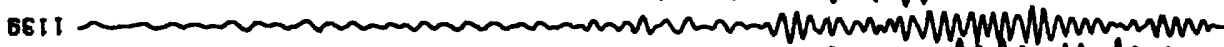

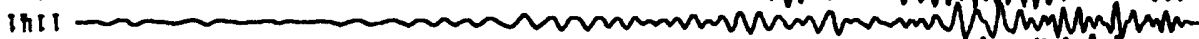
EhII Shtt ind Lhtl i

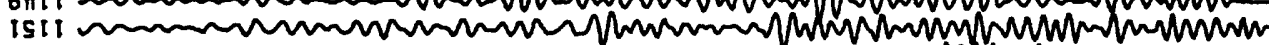

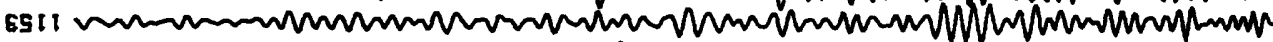
SSII II

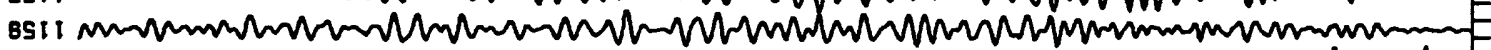
1911 EstI

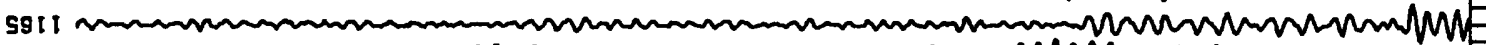

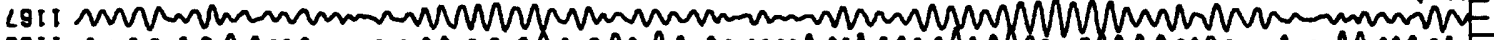
1911 rmand

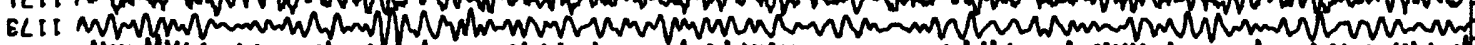

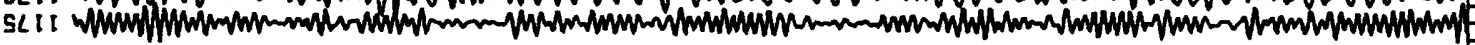

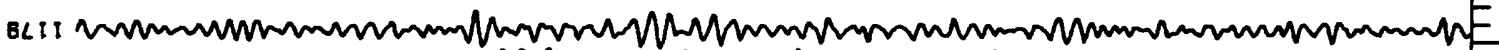

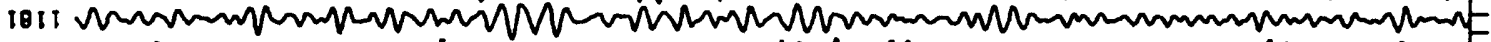

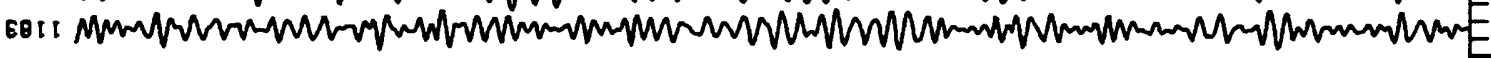
cori Connm

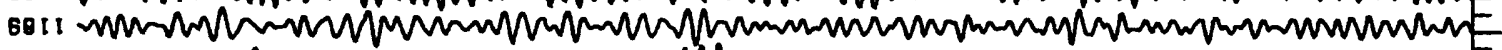

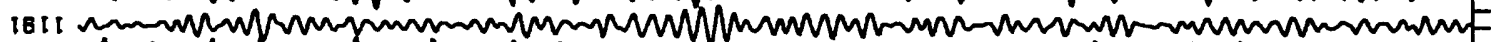

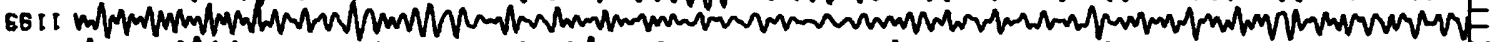

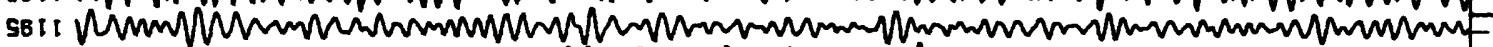

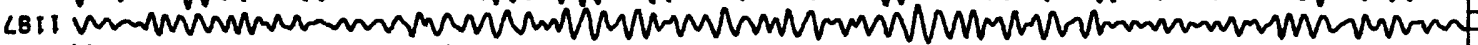
8в 1 Why

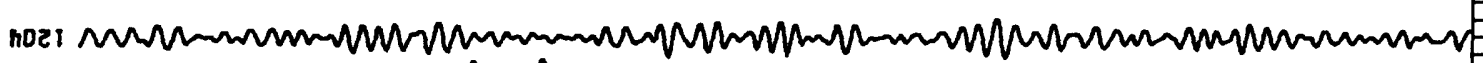
9021 -

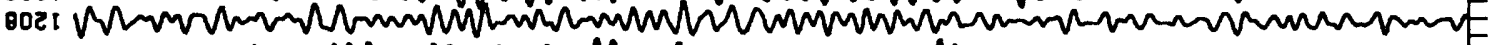

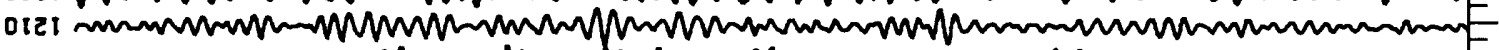

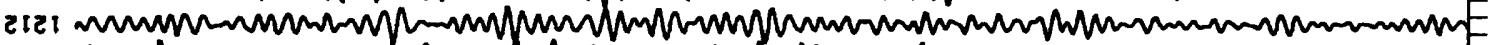

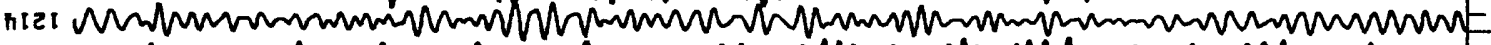

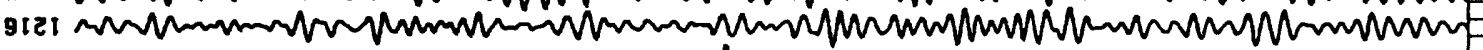

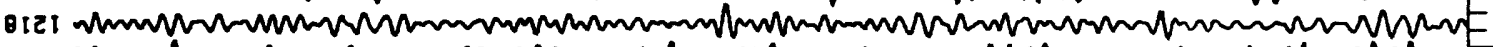

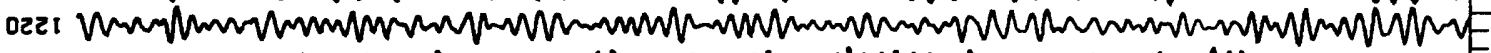

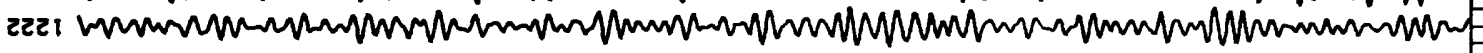


oh

on

oh

on

oh

II SIII

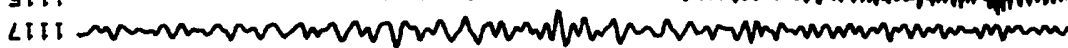
BIII Ifl

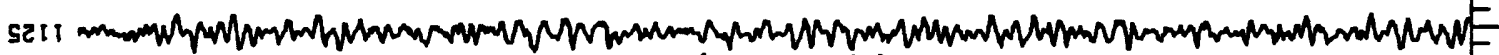
LEII

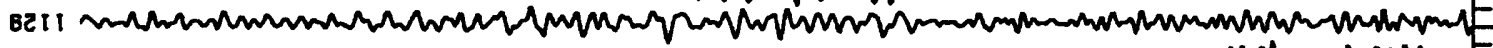
IE IT

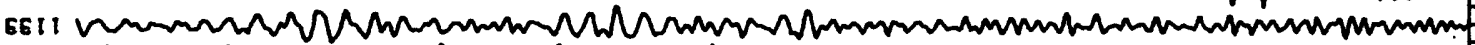

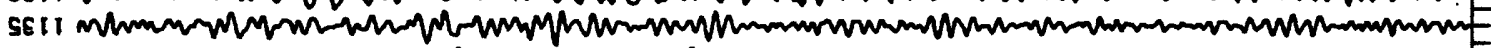

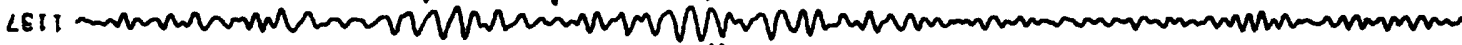

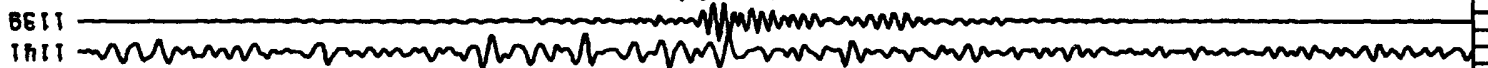

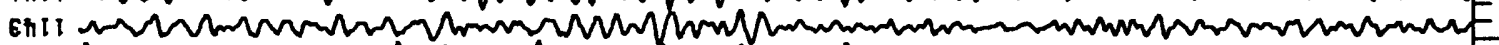

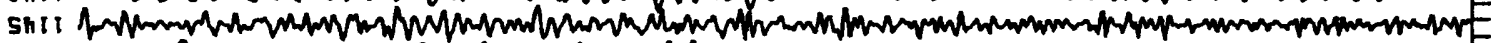

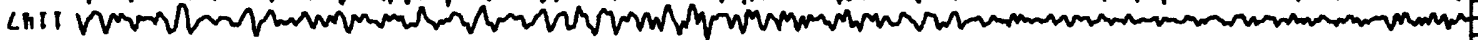
is I I

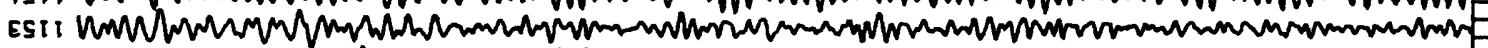

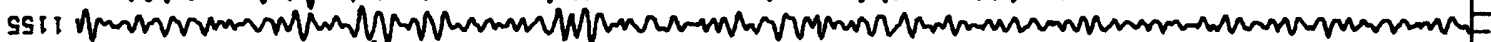

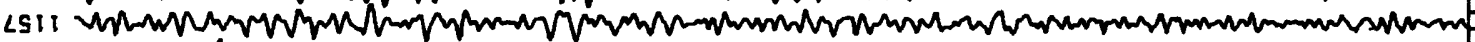

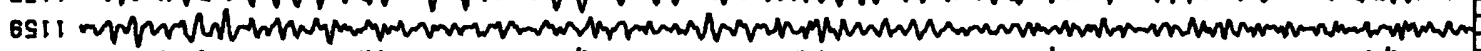

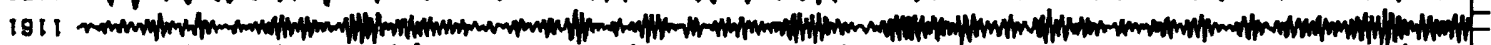

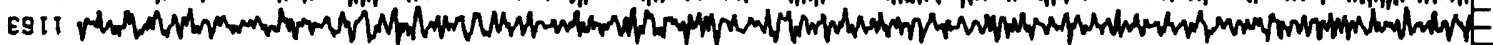

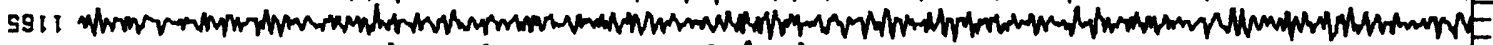

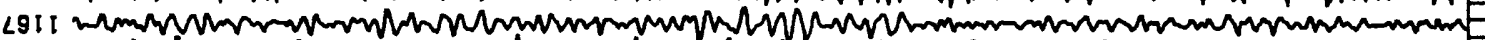
iLI I

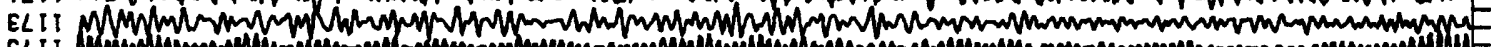

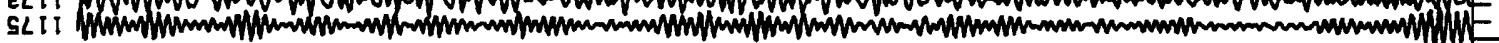

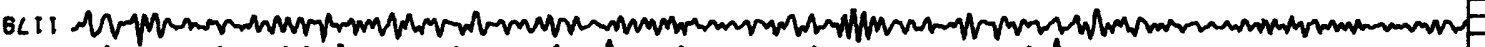

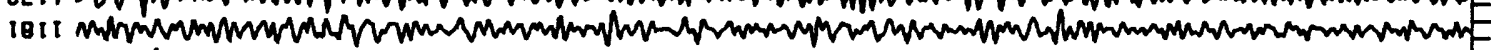

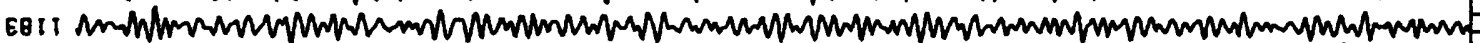

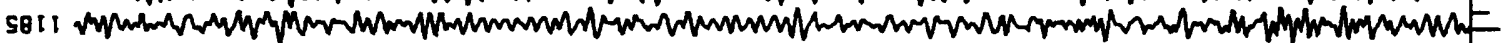

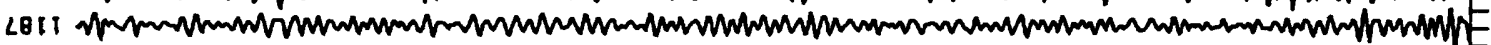

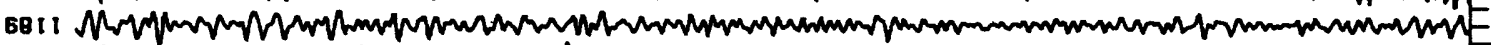

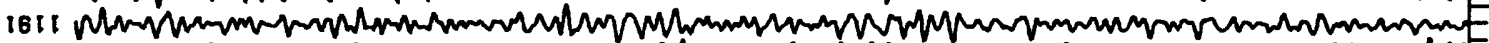

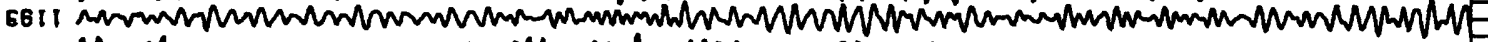

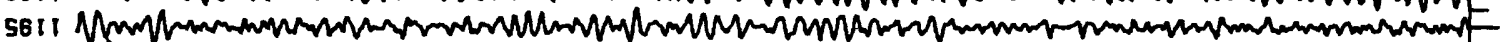

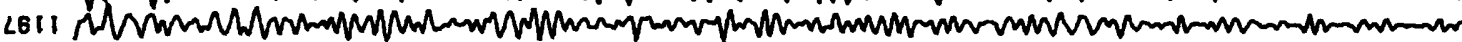

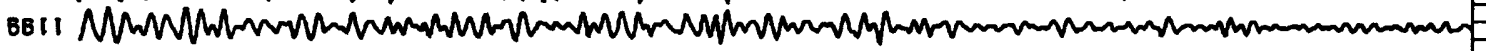

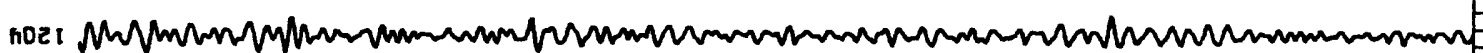

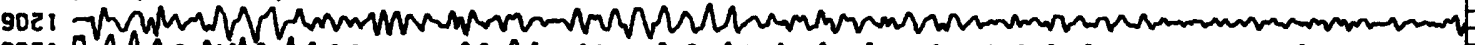

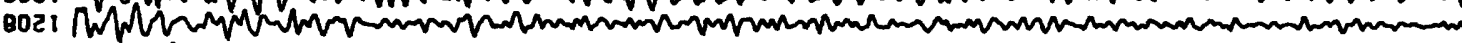

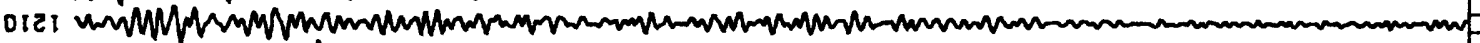

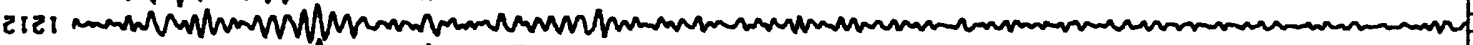

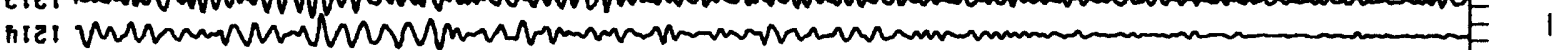

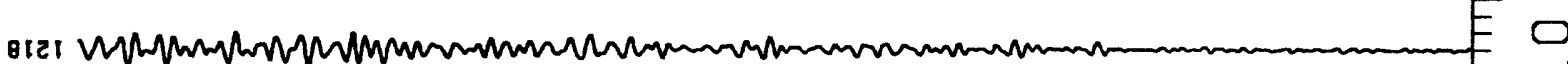

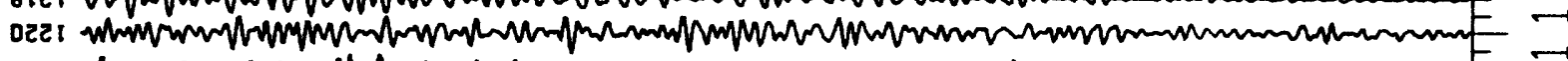

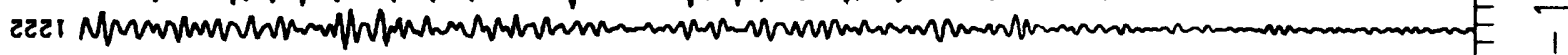

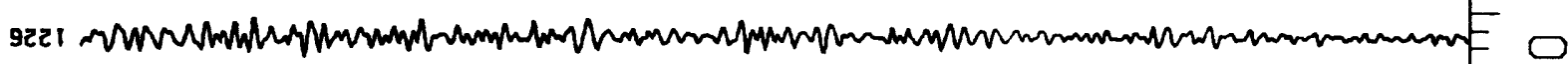

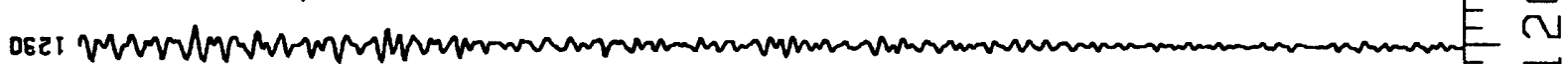

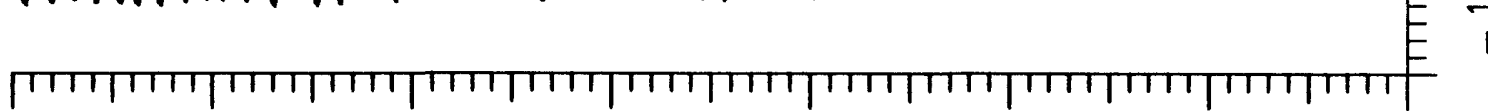




\section{Acknowledgments}

The authors would like to express their appreciation to Dale Stephenson and the Savannah River Technology Center in the Environmental Science section of the Savannah River Company and to the members of the USGS field crew for their assistance in making the field operations successful.

Funding for this work was provided by the U.S. Department of Energy and the U.S. Geological Survey. 


\section{References}

Ackermann, H.D., 1983, Seismic-refraction in the area of the Charleston, South Carolina, 1886 earthquake, in Gohn, G.S., ed., Studies related to the Charleston, South Carolina, earthquake of 1886 - Tectonics and Seismicity, USGS Prof. Paper 1313, F1-F20.

Bonini W.E.and Woollard G.P., 1960, Subsurface Geology of North Carolina-South Carolina Coastal Plain from seismic data : AAPG Bulletin, v.44, p.298315 .

Cook, F.A., L.D. Brown, S. Kaufman, J.E. Oliver, and T.A. Petersen, 1981, COCORP seismic profiling of the Appalachian orogen beneath the Coastal Plain of Georgia: GSA Bull., v.92, p.738-748.

Daniels,D.L., 1974, Geologic interpretation of geophysical maps, central Savannah River area, South Carolina and Georgia: U.S. Geol. Survey Map GP-893, 9 p. text.

Daniels, D.I. and I. Zietz, 1978, Geologic interpretation of aeromagnetic maps of the Coastal plain region of south Carolina and parts of North Carolina and Georgia, USGS Open-File Report 78-261.

Daniels, D.L., I. Zietz, and P. Popenoe, 1983, Distribution of subsurface lower Mesozoic rocks in the southeastern United States as interpreted from regional gravity and magnetic maps, in Gohn, G.S., ed., Studies related to the Charleston, South Carolina, earthquake of $1886-$ Tectonics and Seismicity, USGS Professional Paper 1313, K1-K24.

Hamilton, R.M., J.C. Behrendt, and H.D. Ackermann, 1983, Land multi-channel seismic-reflection evidence for tectonic features near Charleston, South Carolina, in Gohn, G.S., ed., Studies related to the Charleston, South Carolina, earthquake of 1886 - Tectonics and seismicity, USGS Prof. Paper 1313, I1-I18.

Maher, J.C., 1971, Geologic framework and petroleum potential of the Atlantic Coastal Plain and Continental Shelf: U.S. Geol. Survey Prof. Paper 659, $98 \mathrm{p}$.

Popenoe P.and zietz I, 1977, The nature of the geophysical basement beneath the Coastal plain of South carolina and northeastern Georgia, in Rankin, D.W., ed., Studies related to the Charleston, South Carolina, earthquake of 1886 - A preliminary report., USGS Prof. Paper 1028-I, $119-137$ 
Instrument site locations

\begin{tabular}{|c|c|c|c|c|c|c|c|}
\hline Location & $\begin{array}{l}\text { Lat. (N) } \\
\text { deg }\end{array}$ & $\begin{array}{c}\text { Long. (W) } \\
\text { deg }\end{array}$ & $\begin{array}{l}\text { Elev. } \\
\text { meters }\end{array}$ & Location & $\begin{array}{c}\text { Lat. (N) } \\
\text { deg }\end{array}$ & $\begin{array}{l}\text { Long. (W) } \\
\text { deg }\end{array}$ & $\begin{array}{l}\text { Elev. } \\
\text { meters }\end{array}$ \\
\hline 110 & 32.883400 & 80.581091 & 25 & 160 & 33.088783 & 81.070826 & 105 \\
\hline 111 & 32.889436 & 80.588983 & 25 & 161 & 33.092671 & 81.078086 & 67 \\
\hline 112 & 32.895054 & 80.597578 & 30 & 162 & 33.097793 & 81.091211 & 72 \\
\hline 113 & 32.896079 & 80.609376 & 35 & 163 & 33.103368 & 81.096130 & 104 \\
\hline 114 & 32.896606 & 80.621324 & 36 & 164 & 33.111672 & 81.105536 & 82 \\
\hline 115 & 32.897741 & 80.632438 & 59 & 165 & 33.114060 & 81.117511 & 99 \\
\hline 116 & 32.904192 & 80.641509 & 49 & 166 & 33.115966 & 81.127403 & 105 \\
\hline 117 & 32.907154 & 80.650807 & 65 & 167 & 33.122959 & 81.136392 & 102 \\
\hline 118 & 32.909769 & 80.663264 & 11 & 168 & 33.129357 & 81.147340 & 102 \\
\hline 119 & 32.913475 & 80.675756 & 57 & 169 & 33.126978 & 81.154218 & 103 \\
\hline 120 & 32.920717 & 80.683719 & 55 & 170 & 33.127438 & 81.161219 & 101 \\
\hline 121 & 32.924592 & 80.691917 & 52 & 171 & 33.123196 & 81.170689 & 91 \\
\hline 122 & 32.932614 & 80.700860 & 56 & 172 & 33.126489 & 81.175836 & 97 \\
\hline 123 & 32.930509 & 80.712613 & 45 & 173 & 33.130811 & 81.185695 & 187 \\
\hline 124 & 32.938187 & 80.724308 & 19 & 174 & 33.139431 & 81.188840 & 121 \\
\hline 125 & 32.937241 & 80.732581 & 30 & 175 & 33.149989 & 81.192303 & 87 \\
\hline 126 & 32.943498 & 80.742861 & 40 & 176 & 33.157068 & 81.202158 & 119 \\
\hline 127 & 32.945099 & 80.756315 & 42 & 177 & 33.163401 & 81.210910 & 119 \\
\hline 128 & 32.945591 & 80.767158 & 40 & 178 & 33.166228 & 81.221614 & 124 \\
\hline 129 & 32.949869 & 80.777051 & 60 & 179 & 33.170399 & 81.231774 & 69 \\
\hline 130 & 32.953098 & 80.787577 & 49 & 180 & 33.174863 & 81.240932 & 126 \\
\hline 131 & 32.958870 & 80.797151 & 62 & 181 & 33.179215 & 81.249789 & 58 \\
\hline 132 & 32.964606 & 80.806439 & 63 & 182 & 33.184031 & 81.259672 & 43 \\
\hline 133 & 32.970649 & 80.817265 & 51 & 183 & 33.189603 & 81.270341 & 62 \\
\hline 134 & 32.972128 & 80.827440 & 74 & 184 & 33.194279 & 81.278356 & 150 \\
\hline 135 & 32.974238 & 80.837758 & 73 & 185 & 33.199330 & 81.288351 & 141 \\
\hline 136 & 32.977325 & 80.847134 & 50 & 186 & 33.204116 & 81.297412 & 131 \\
\hline 137 & 32.983371 & 80.858191 & 44 & 187 & 33.209830 & 81.307568 & 117 \\
\hline 138 & 32.988705 & 80.868518 & 61 & 188 & 33.212590 & 81.317539 & 129 \\
\hline 139 & 32.993038 & 80.880233 & 61 & 189 & 33.216005 & 81.329156 & 149 \\
\hline 140 & 32.995484 & 80.888669 & 58 & 190 & 33.221750 & 81.338819 & 142 \\
\hline 141 & 32.999793 & 80.898341 & 49 & 191 & 33.2 & 81.350005 & 155 \\
\hline 142 & 33.007060 & 80.907377 & 61 & 192 & 33.228594 & 81.357411 & 80 \\
\hline 143 & 33.008466 & 80.917602 & 35 & 193 & 33.236772 & 81.364773 & 48 \\
\hline 144 & 33.019162 & 80.925821 & 65 & 194 & 33.238026 & 81.375948 & 38 \\
\hline 145 & 33.024227 & 80.932036 & 62 & 195 & 33.242788 & 81.385791 & 107 \\
\hline 146 & 33.029269 & 80.938620 & 62 & 196 & 33.244274 & 81.397415 & 105 \\
\hline 147 & 33.031726 & 80.949410 & 62 & 197 & 33.252416 & 81.403761 & 145 \\
\hline 148 & 33.028473 & 80.967121 & 73 & 198 & 33.246774 & 81.419228 & 121 \\
\hline 149 & 33.035611 & 80.973471 & 77 & 199 & 33.257264 & 81.428930 & 127 \\
\hline 150 & 33.045149 & 80.973417 & 69 & 200 & (no site) & & \\
\hline 151 & 33.055062 & 80.976596 & 66 & 201 & 33.266717 & 81.440301 & 77 \\
\hline 152 & 33.063788 & 80.984287 & 69 & 202 & 33.268225 & 81.446338 & 110 \\
\hline 153 & 33.063992 & 80.994731 & 55 & 203 & 33.273579 & 81.454501 & 109 \\
\hline 154 & 33.069392 & 81.002053 & 76 & 204 & 33.276915 & 81.465588 & 109 \\
\hline 155 & 33.078513 & 81.011119 & 81 & 205 & 33.281019 & 81.477419 & 141 \\
\hline 156 & 33.078356 & 81.023643 & 98 & 206 & 33.289057 & 81.486578 & 144 \\
\hline 157 & 33.074619 & 81.038514 & 81 & 207 & 33.293681 & 81.492861 & 124 \\
\hline 158 & 33.084649 & 81.048372 & 34 & 208 & 33.299344 & 81.500748 & 122 \\
\hline 159 & 33.086960 & 81.056079 & 54 & 209 & 33.304084 & 81.510528 & 152 \\
\hline
\end{tabular}


Appendix A

$\begin{array}{rcclcccr}\text { Location } & \begin{array}{c}\text { Lat. (N) } \\ \text { deg }\end{array} & \begin{array}{c}\text { Long. (W) } \\ \text { deg }\end{array} & \begin{array}{c}\text { Elev. } \\ \text { meters }\end{array} & \begin{array}{c}\text { Location } \\ \text { Lat. (N) } \\ \text { deg }\end{array} & \begin{array}{c}\text { Long. (W) } \\ \text { deg }\end{array} & \begin{array}{c}\text { Elev. } \\ \text { meters }\end{array} \\ 210 & 33.307467 & \mathbf{8 1 . 5 2 1 2 9 6} & 147 & 220 & 33.350218 & \mathbf{8 1 . 6 2 1 4 8 5} & 110 \\ 211 & 33.312207 & \mathbf{8 1 . 5 3 0 4 9 0} & 159 & 221 & 33.352386 & \mathbf{8 1 . 6 3 2 9 0 8} & \mathbf{7 9} \\ 212 & 33.317465 & \mathbf{8 1 . 5 4 1 2 0 9} & 148 & \mathbf{2 2 2} & 33.365649 & \mathbf{8 1 . 6 3 9 8 7 2} & \mathbf{9 3} \\ 213 & 33.322359 & \mathbf{8 1 . 5 5 2 2 3 8} & 148 & \mathbf{2 2 3} & 33.377662 & \mathbf{8 1 . 6 4 0 4 8 1} & 102 \\ 214 & 33.320916 & \mathbf{8 1 . 5 6 3 2 3 3} & 146 & \mathbf{2 2 4} & 33.380663 & \mathbf{8 1 . 6 5 3 2 2 5} & 166 \\ 215 & 33.322255 & \mathbf{8 1 . 5 7 4 6 3 9} & 134 & 225 & 33.384401 & \mathbf{8 1 . 6 6 4 4 7 9} & 197 \\ 216 & 33.329084 & \mathbf{8 1 . 5 8 3 1 1 9} & 141 & \mathbf{2 2 6} & 33.388150 & \mathbf{8 1 . 6 7 5 4 2 1} & 198 \\ 217 & 33.332832 & \mathbf{8 1 . 5 9 5 5 3 3} & 151 & \mathbf{2 2 7} & 33.391101 & \mathbf{8 1 . 6 8 3 7 4 6} & 197 \\ 218 & 33.337333 & \mathbf{8 1 . 6 0 5 0 3 3} & 104 & \mathbf{2 2 8} & 33.394683 & \mathbf{8 1 . 6 9 3 9 9 0} & 157 \\ 219 & 33.344673 & \mathbf{8 1 . 6 1 1 3 1 8} & 107 & 229 & 33.406338 & \mathbf{8 1 . 6 9 8 8 2 6} & 166 \\ & & & & 230 & 33.410521 & \mathbf{8 1 . 7 0 6 1 9 6} & 171\end{array}$




\section{Appendix B - SEGY Data File Format}

The data from this experiment are archived in an extended version of the standard SEGY seismic data format. Data is organized by shotgathers; one SEGY file per shotpoint. SEGY data files are sequentially written to tape with intervening EndOf-File marks.

c

c

\section{INCLUDE FILE FOR FORTRAN PROGRAMS TO READ SEGY DATA FILES}

c

c This file is an implicit definition of SEGY format with additions

c for refraction work. It is the SEGY standard of Barry et al

c Geophysics (1975) with extensions labelled LDS USE and USGS use

c for refraction work. When used as an include file for a FORTRAN

c program, all variables will be set after reading arrays SEGY1A,

c SEGY1B, and SEGYDB.

c

c Character code is EBCDIC unless IEEE data format (see variable icode)

c If IEEE, then the character code is ASCII.

c

c Written by Carl Spencer and Isa Asudeh 4/2/86 original specification

c This version is compatible with the final Lithoprobe version dated 5/12/87.

c

c Maximum number of bytes allowed in a trace (system dependent)

c $\operatorname{MAXLEN}=((\max$ trace length $) *($ sample rate $) *($ bytes per sample $))+240$

c

parameter (MAXLEN=16620)

c

c

c SEGY REEL IDENTIFICATION HEADER PART 1 byte segy $1 \mathrm{a}(3200)$

c SEGY REEL IDENTIFICATION HEADER PART 2 byte segylb(400)

c SEGY TRACE DATA BLOCK byte segydb(MAXLEN) common/segycom/iiopen,segy $1 \mathrm{a}$, segy 1 b, segydb

c

c EBCDIC CARDS

character*80 cards(40)

equivalence (segy 1a(1),cards(1))

c

c TRACE IDENTIFICATION HEADER

byte thead(240)

equivalence (segydb(1),thead(1))

C

c DATA WORDS

integer*2 iidata((MAXLEN-240)/2)

integer*4 jdata((MAXLEN-240)/4)

real*4 rdata((MAXLEN-240)/4)

equivalence (segydb(241),iidata(1),jdata(1), rdata(1))

c 


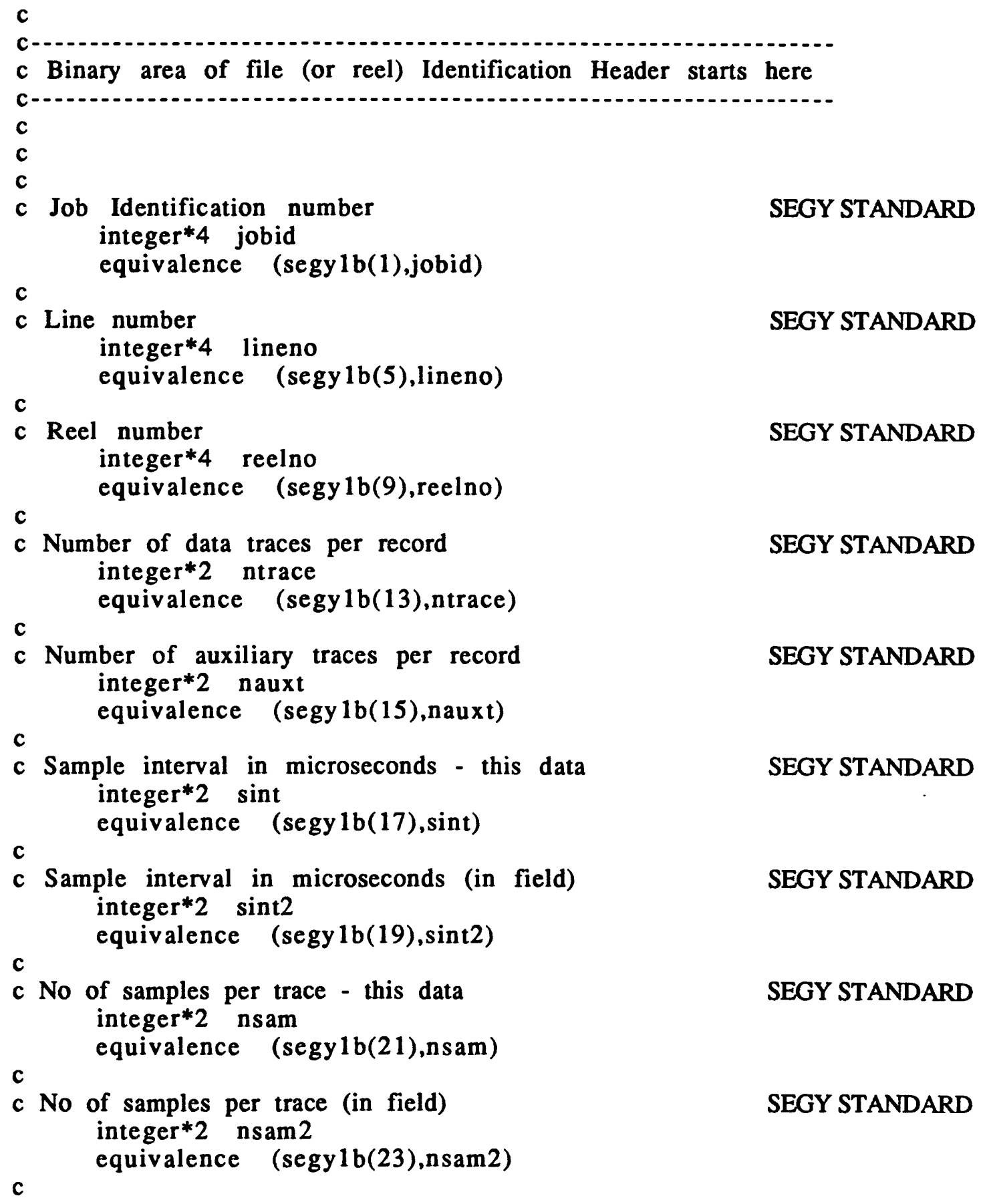

c Sample interval in microseconds (in field) integer*2 sint 2 
c Data sample format code

c icode $=0001$

c icode $=0002$

c icode $=0003$

c icode $=0004$

c icode $=0100$

c icode $=0200$

c icode $=0300$

c icode $=0500$

(1)

(2)

(3)

(4)

(256)

(512)

(768)

(1280)

(1536)

integer*2 icode

equivalence (segy1b(25),icode)

c

c Number of traces in CDP ensemble integer*2 ncdp

equivalence (segy $1 b(27), n c d p)$

c

c Trace sorting code

c itsort $=1$ as recorded

c itsort $=2$ CDP ensemble

c itsort $=3$ Single fold continuous

c itsort $=4$ Horizontal stack

c No LDS or USGS use. integer*2 itsort equivalence (segylb(29),itsort)

c

c Vertical sum code

c vcode $=\mathrm{n}$ sum on $\mathrm{n}$ traces

integer*2 vcode

equivalence (segy1b(31),vcode)

c

c Start sweep frequency (hz)

integer*2 ssweep

equivalence (segy1b(33),ssweep)

c

c End sweep frequency (hz)

integer*2 esweep

equivalence (segy $1 b(35)$, esweep)

c

c Sweep length in milliseconds

integer*2 sleng

equivalence (segy1b(37),sleng)

c

c Sweep type

c stype $=1$ Linear

c stype $=2$ Parabolic

c stype $=3$ Exponential

c stype $=4$ Other

c stype $=5$ Borehole source

c stype $=6$ Water explosive source

c stype $=7$ Airgun source

integer*2 stype

equivalence (segy1b(39),stype)
SEGY STANDARD

SEGY STANDARD

SEGY STANDARD

SEGY STANDARD

SEGY STANDARD

VERITAS STANDARD

LDS USE

LDS USE

SEGY STANDARD

SEGY STANDARD

SEGY STANDARD

SEGY STANDARD

SEGY STANDARD

SEGY STANDARD

SEGY STANDARD

SEGY STANDARD

SEGY STANDARD

SEGY STANDARD

SEGY STANDARD

SEGY STANDARD

SEGY STANDARD

SEGY STANDARD

SEGY STANDARD

LDS USE

LDS USE

LDS USE 


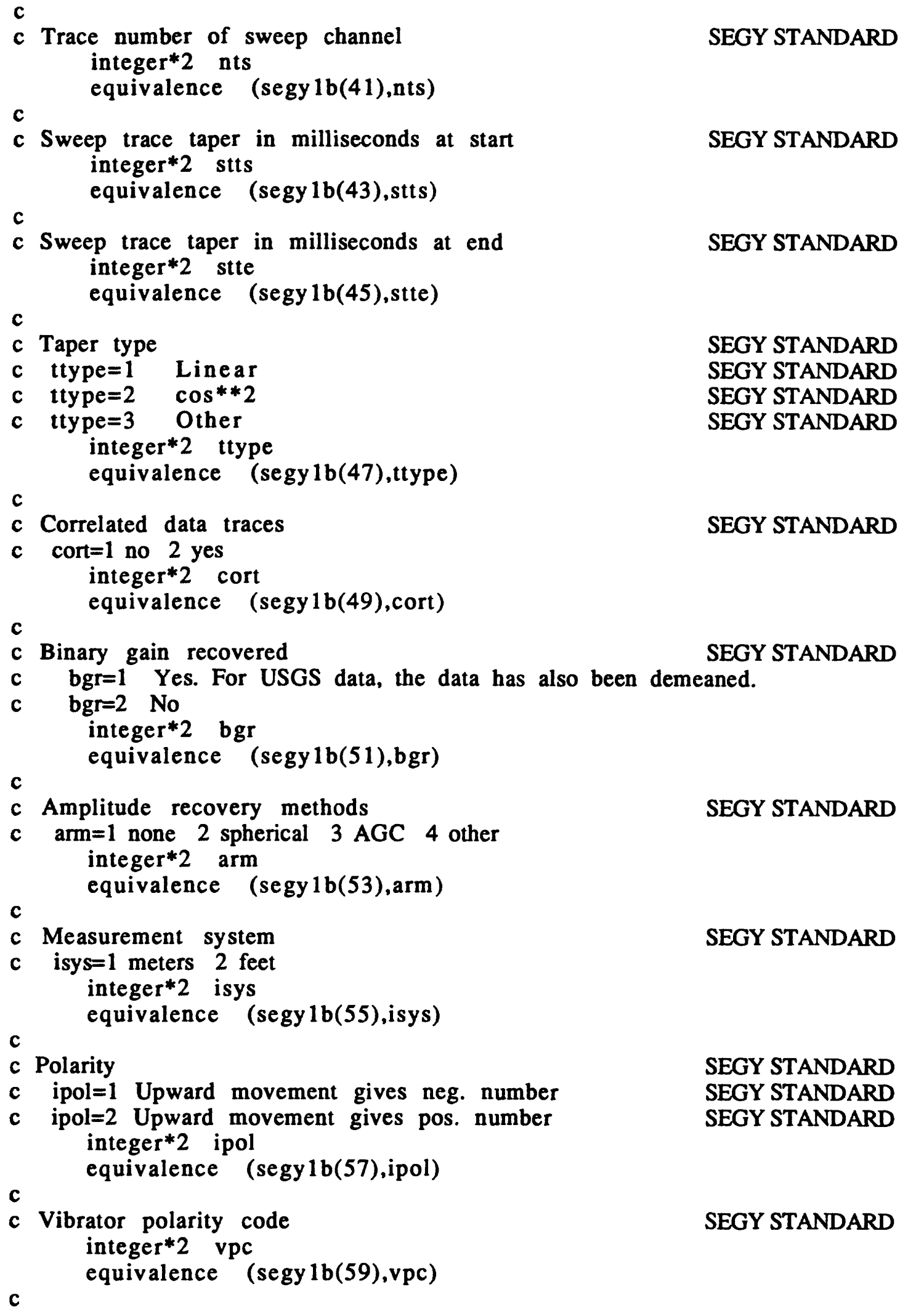


c Number of traces in the file

LDS USE

c Used for disk files. integer*2 notif equivalence (segy $1 b(61)$, notif)

c

c Attribute information

c attri=0 velocity/displacement data

c attri $=1$ instantaneous amplitude

c attri $=2$ instantaneous frequency

c attri=3 instantaneous phase

c attri $=4$ slowness $(\mathrm{m} / \mathrm{ms})$

c attri=5 semblance $(0-1000)$ integer*2 attri

equivalence (segy1b(63), attri)

c

c Mean amplitude of all samples

c in all traces in file Used for disk files. real*4 meanas equivalence (segy1b(65),meanas)

c

c Domain of data

domain $=0$ Time - distance domain domain=1 Frequency - wavenumber domain domain=2 Intercept time - slowness domain integer*2 domain equivalence (segylb(69),domain)

c

c Bytes 71, 72 unused to align four byte boundaries.

c

c Reduction velocity meters/sec if data is reduced integer*4 vred equivalence (segy $1 b(73), v$ red)

c

c Minimum of all samples in file. real*4 minass equivalence (segy $1 \mathrm{~b}(77)$,minass)

c

c Maximum of all samples in file. real*4 maxass equivalence (segy $1 b(81)$, maxass)

c

c Recording instrument type

c iinstr $=1$ EDA lunchbox recorder

c iinstr $=2$ USGS seismic cassette recorder

c iinstr=3 GBOS

c iinstr $=99$ Mixed integer*2 iinstr

equivalence (segy $1 \mathrm{~b}(85)$,iinstr)

c

c File creation date - Last two digits of year integer*2 cryear equivalence (segy $1 b(87)$,cryear)

c

LDS USE

LDS USE

LDS USE

LDS USE

LDS USE

LDS USE

USGS USE

USGS USE 
c File creation date - Month of year

USGS USE

integer*2 crmnth

equivalence (segy $1 \mathrm{~b}(89), \mathrm{crmnth})$

c

c File creation date - Day of month integer*2 crday

equivalence (segy $1 b(91), c r d a y)$

c

Bytes 93-398 of the binary File Identification Header are not used

c

c Format version number (x100)

c Version 0.99 "Discussion version", October 1986.

c Version 1.00 "Final version", December 5, 1987 integer*2 fvn

equivalence (segy $1 \mathrm{~b}(399), \mathrm{fvn})$

c

USGS USE 


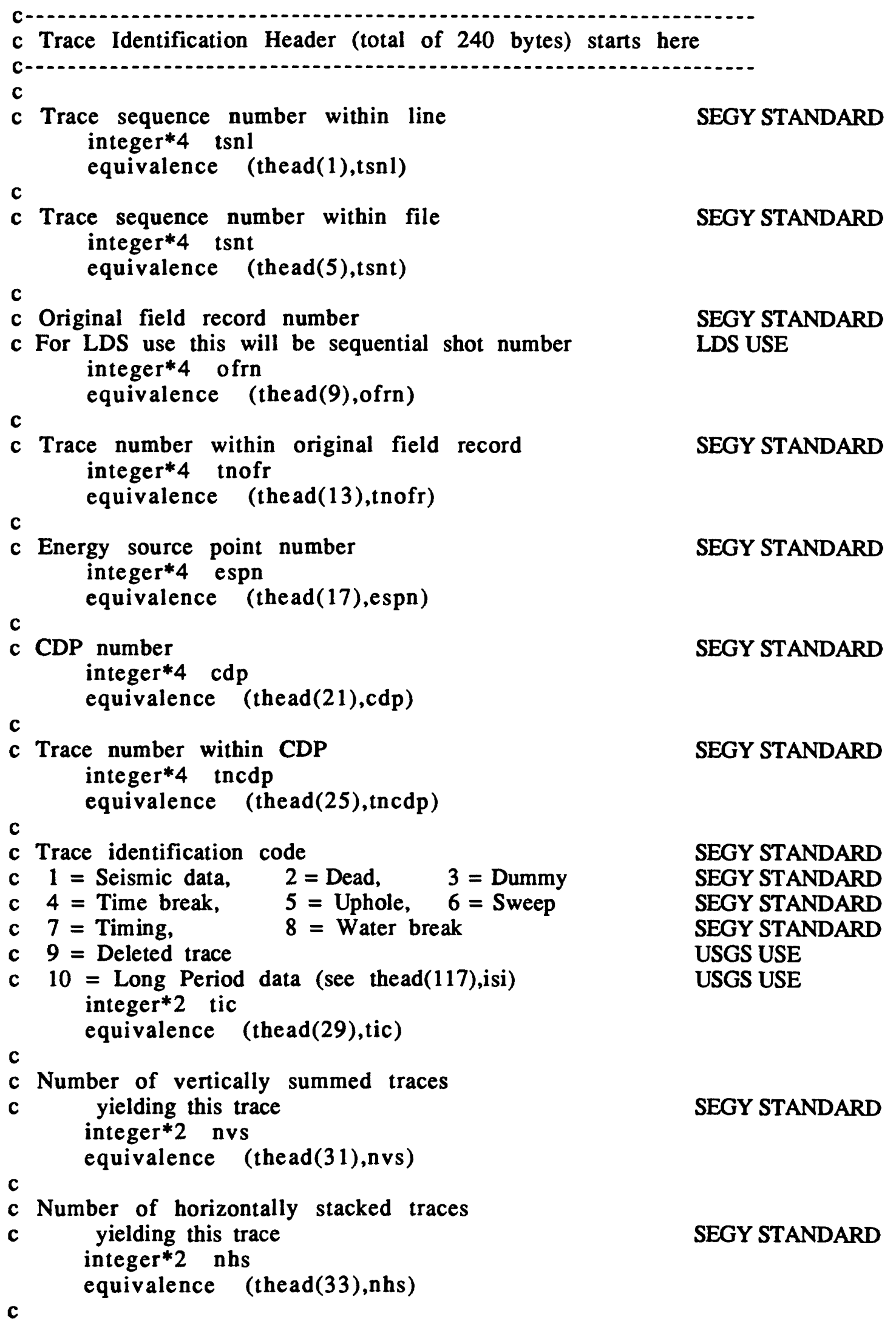


c Data use (1=production $2=$ test)

SEGY STANDARD integer*2 duse equivalence (thead(35),duse)

c

c Distance from source to receiver integer*4 idist equivalence (thead(37),idist)

SEGY STANDARD

C

c Receiver group elevation integer*4 irel equivalence (thead(41),irel)

SEGY STANDARD

c

c Surface elevation of source integer*4 ishe equivalence (thead(45),ishe)

SEGY STANDARD

c

c Shot depth integer*4 ishd equivalence (thead(49),ishd)

SEGY STANDARD

c

c Datum elevation at receiver integer*4 delr equivalence (thead(53),delr)

SEGY STANDARD

c

c Datum elevation at source integer*4 dels equivalence (thead(57),dels)

SEGY STANDARD

c

c Water depth at source

SEGY STANDARD integer*4 wds equivalence (thead(61),wds)

c

c Water depth at receiver integer*4 wdr equivalence (thead $(65), w d r)$

c

c Scalar multiplier/divisor for bytes 41-68 integer*2 smul1 equivalence (thead(69),smul1)

c

c Scalar multiplier/divisor for bytes $73-88$ integer*2 smul2 equivalence (thead(71),smul2)

c

c Source coordinate $X$ or longitude (East positive) integer*4 ishlo equivalence (thead(73),ishlo)

c

c Source coordinate $Y$ or latitude (North positive) integer*4 ishla equivalence (thead(77),ishla)

c

SEGY STANDARD

SEGY STANDARD

SEGY STANDARD

SEGY STANDARD

SEGY STANDARD 


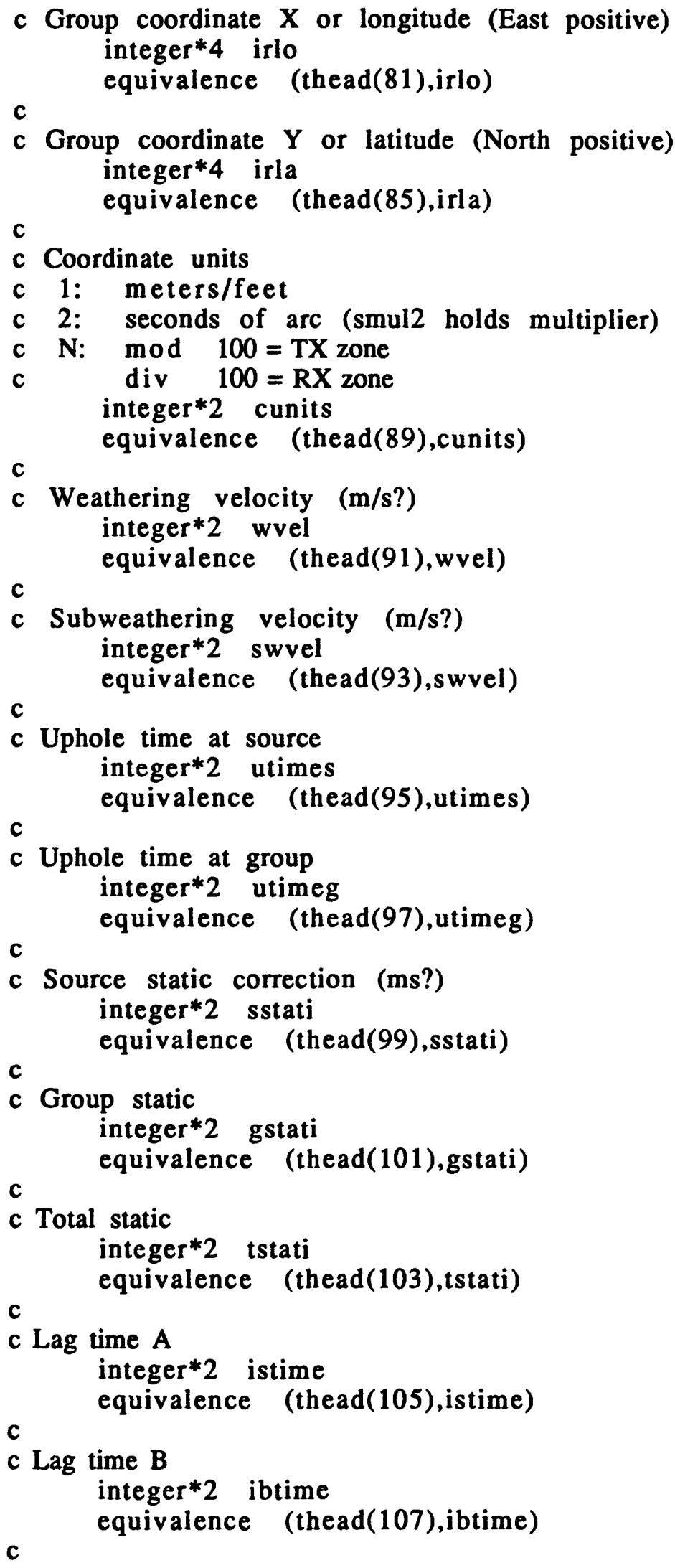

integer*2 ibtime

SEGY STANDARD

SEGY STANDARD

SEGY STANDARD

SEGY STANDARD

SEGY STANDARD

SEGY STANDARD

SEGY STANDARD

SEGY STANDARD

SEGY STANDARD

SEGY STANDARD

SEGY STANDARD

SEGY STANDARD 


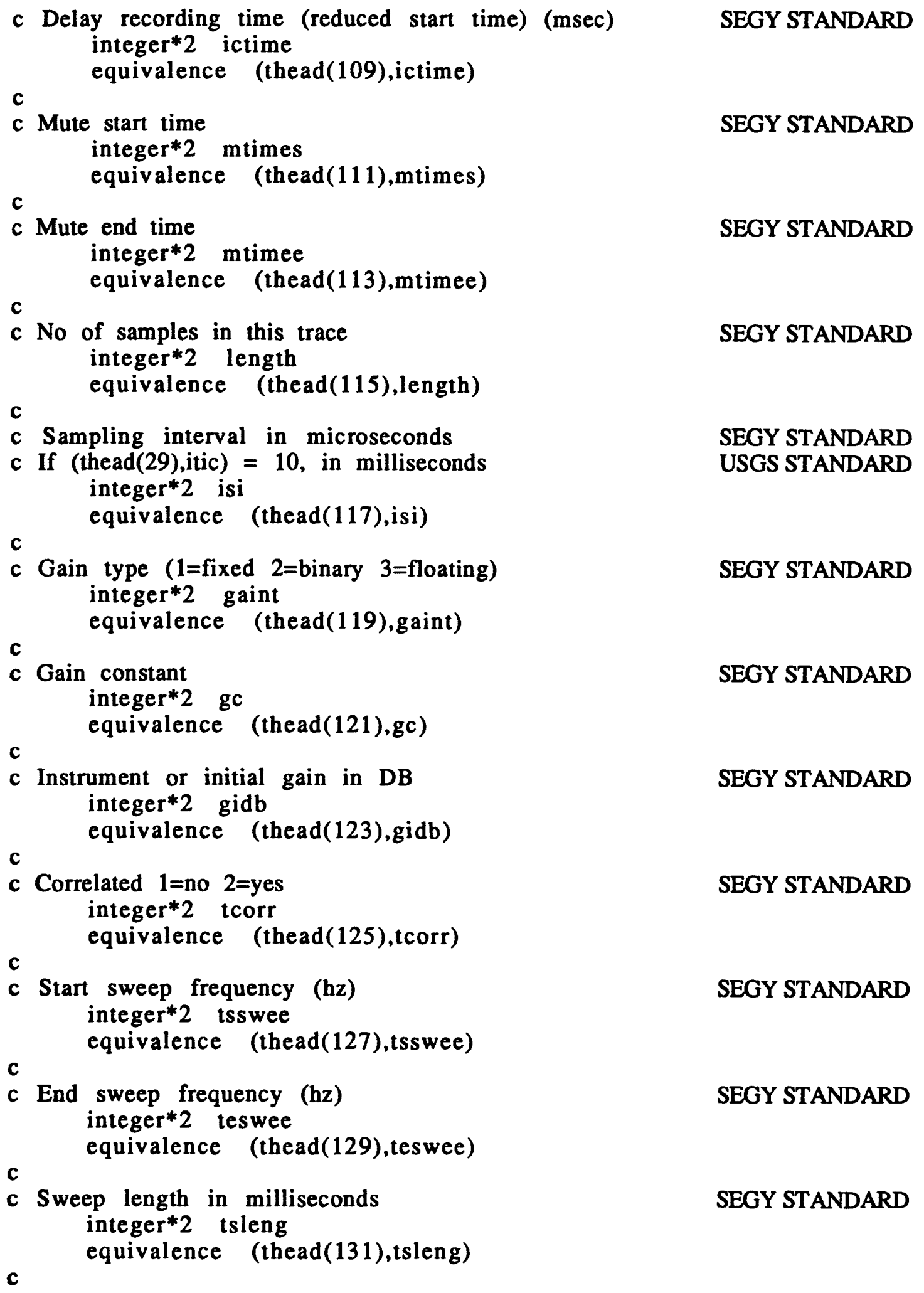


c Sweep type

c stype $=1$

c stype $=2$

c stype $=3$

c stype $=4$

c stype $=5$

c stype $=6$

c stype $=7$ integer*2

Linear

Parabolic

Exponential

Other

Borehole source

Water explosive source

Airgun source

equivalence (thead(133),tstype)

c

c Sweep trace taper in milliseconds at start integer*2 tstts equivalence (thead(135),tstts)

c

c Sweep trace taper in milliseconds at end integer*2 tstte equivalence (thead(137),tstte)

c

c Taper type

c ttype $=1$ Linear

c ttype $=2$ Cos**2

c ttype $=3$ Other integer*2 tttype

equivalence (thead(139),tttype)

c

c Antialias filter frequency

integer*2 aif

equivalence (thead(141), aif)

c

c Alias filter slope integer*2 ais equivalence (thead(143),ais)

c

c Notch filter frequency integer*2 nif equivalence (thead(145),nif)

C

c Notch filter slope integer*2 nis equivalence (thead(147),nis)

c

c Low cut frequency integer*2 flc equivalence (thead(149),flc)

c

c High cut frequency integer*2 fhc equivalence (thead(151),fhc)

c

c Low cut slope integer*2 slc equivalence (thead $(153)$, slc)
SEGY STANDARD

SEGY STANDARD

SEGY STANDARD

SEGY STANDARD

SEGY STANDARD

LDS USE

LDS USE

LDS USE

SEGY STANDARD

SEGY STANDARD

SEGY STANDARD

SEGY STANDARD

SEGY STANDARD

SEGY STANDARD

SEGY STANDARD

SEGY STANDARD

SEGY STANDARD

SEGY STANDARD

SEGY STANDARD

SEGY STANDARD

SEGY STANDARD 
c

c High cut slope integer*2 shc equivalence (thead(155),shc)

c

c Year of start of trace integer*2 tyear

SEGY STANDARD equivalence (thead(157),tyear)

SEGY STANDARD

c

c Day of start of trace

integer*2 tday

SEGY STANDARD

equivalence (thead(159),tday)

c

c Hour of start of trace integer*2 thour equivalence (thead(161),thour)

c

c Minute of start of trace integer*2 tmin equivalence (thead(163),tmin)

c

c Second of start of trace integer*2 tsec equivalence (thead(165),tsec)

SEGY STANDARD

SEGY STANDARD

c Time basis code $1=$ local $2=\mathrm{GMT}$ integer*2 tbcode equivalence (thead(167),tbcode)

SEGY STANDARD

c

c Trace weighting factor integer*2 twf equivalence (thead(169),twf)

c

c Geophone group no. on roll switch first position integer*2 ggrp1 equivalence (thead(171),ggrp1)

c

c Geophone group no. trace position 1

c on field record integer*2 ggtp equivalence (thead(173),ggtp)

c

c Time code translator error light

SEGY STANDARD

SEGY STANDARD

c $1=$ No error $2=$ Error integer*2 errlt

c equivalence (thead(175),errlt)

SEGY STANDARD

SEGY STANDARD

USGS USE

Distance-azimuth calculation algorithm

USGS USE

1 = Sodano algorithm. The program utilizes the Sodano and Robinson (1963) direct solution of geodesics (Army Map Service, Tech Rep \#7, Section IV). integer*2 daca equivalence (thead(177),daca) 
C

c Earth dimension code

c 1 = Fischer spheroid (1960),

c OMEGA \& NASA datums

c 2 = Clark ellipsoid (1866),

c N. American datum 1927

c $3=$ Ref ellipsoid (1967), S. American datum

c $4=$ Hayford International Ellipsoid (1910)

c $5=$ World Geodetic Survey Ellipsoid (1972)

c $6=$ Bessel (1841), Tokyo datum

c 7 = Everest (1830), India datum

c 8 = Airy (1936),

c Ordnance survey of Great Britain

c 9 = Hough (1960), Wake-Eniwetok

c $10=$ Fischer (1968), Modified Mercury

c 11 = Clarke (1880) integer* 2 edc equivalence (thead(179),edc)

c

c Microseconds of trace start time integer*4 mst equivalence (thead(181),mst)

c

c Millisecond of timing correction integer*2 cor equivalence (thead(185),cor)

.

c Charge size in $\mathbf{~ k g}$ integer*2 charge equivalence (thead(187),charge)

.

c Shot time - Year integer*2 syear equivalence (thead(189), syear)

c

c Shot time - Day integer*2 sday equivalence (thead(191),sday)

c

c Shot time - Hour integer*2 shour equivalence (thead(193),shour)

c

c Shot time - Minute integer*2 shmin equivalence (thead(195),shmin)

c

c Shot time - Second integer*2 sseco equivalence (thead(197),sseco)

\section{USGS USE}

6378166.

298.30

$6378206.4 \quad 294.98$

$6378160 \quad 298.25$

$6378388 . \quad 297.00$

6378135.298 .26

$6377397 . \quad 299.15$

6377276 . $\quad 300.80$

$6377563 . \quad 299.32$

$6378270 . \quad 297.00$

$6378150 . \quad 298.30$

6378249 . 293.47

LDS USE

LDS USE

LDS USE

LDS USE

LDS USE

LDS USE

LDS USE

LDS USE

c 
c Shot time - Microsecond integer*4 ssmic equivalence (thead(199),ssmic)

C

c Azimuth of receiver from shot (minutes of arc) integer*2 azimut equivalence (thead(203), azimut)

c

c Azimuth of geophone orientation axis with

c respect to true north in minutes of arc integer*2 geoazi

equivalence (thead(205),geoazi)

c

c Angle between geophone orientation axis and

c vertical in minutes of arc integer*2 geover equivalence (thead(207),geover)

C

c Time to be added to recorded trace time to get

c actual trace start time. To be used when data

c has been reduced but start time is not updated

c so that the actual time can be recovered even if

c distance and shot time have changed

c (microseconds)

integer*4 ttrace

equivalence (thead(209),ttrace)

c

c Recording instrument number character*4 scrs equivalence (thead(213),scrs)

C

c Deployment name character*4 deploy equivalence (thead(217),deploy)

c

c Shotpoint name (shotpoint number) character*4 spname equivalence (thead(221),spname)

c

c Receiver site name (station number) character*4 rstnam equivalence (thead(225),rstnam)

c

c Shot name (shot number) character*4 shotid equivalence (thead(229), shotid)

c

LDS USE

LDS USE

LDS USE

LDS USE

LDS USE

LDS USE

LDS USE

LDS USE

LDS USE

LDS USE 
c Line name character*4 lineid

equivalence (thead(233), lineid)

c

c Geophone orientation eg R40,Z character*4 geoor equivalence (thead(237),geoor)

c

c End of Trace Identification Header
LDS USE

LDS USE 